\title{
A "spherical shell number density" model for violently relaxed $N$-body systems
}

\author{
C. Efthymiopoulos and N. Voglis
}

\author{
Research Center for Astronomy, Academy of Athens, Anagnostopoulou 10-14, Athens, Greece \\ e-mail: cefthim@cc.uoa.gr; nvogl@cc.uoa.gr
}

Received 18 April 2001 / Accepted 24 July 2001

\begin{abstract}
We present the results of a series of numerical simulations of gravitational collisionless $N$-body systems in equilibrium after a violent relaxation from cosmological initial conditions. The distribution function $f$ of such systems has a complicated form due to the complex structure of the phase space of stellar orbits. This complexity makes hardly tractable the old problem of writing a simple model for $f$. However, we show that it is possible to benefit from various statistical regularities of the phase space in order to compose a heuristic approximation for $f$. Such regularities are revealed if we decompose a system in a number of spherical shells. For each shell we define thermodynamical quantities (e.g., temperatures) which, as we find, vary smoothly with the radius $r$ of the shell. Using these quantities, we find a model that fits the number density function $\nu\left(\mathcal{E}, L^{2}, r\right)$ in each shell. For the greatest range of energies, this function tends to the form of the Stiavelli-Bertin (1987) model. By adding the contributions of all the spherical shells, we then find a global model for $f$. While our method is based on a spherical approximation, we show that it reproduces very accurately the global profiles of our triaxial $N$-body systems.
\end{abstract}

Key words. galaxies: formation - galaxies: kinematics and dynamics - stellar dynamics

\section{Introduction}

The form of the distribution function $f(x, p)$ that describes self-consistently the equilibrium state of a triaxial stellar system is a particularly difficult problem. The difficulty arises from the fact that neither the number nor the form of the integrals of motion for different sets of orbits are known. Numerical studies of 3D systems have shown that for the most interesting range of energies of stellar orbits, the phase space is composed of chaotic zones interwoven with regular ones in a very complicated structure. The resonant structure of the "Arnold web" has been studied in galactic 3D hamiltonians by means of frequency analysis methods (e.g., Papaphilippou \& Laskar 1998; see Merritt 1999 for a review). Such studies reveal that the motion is regular along some resonances and chaotic along other ones. Ordered orbits must obey three integrals of the motion, yet the form of these integrals is different for each different resonance. On the other hand, chaotic orbits obey fewer than three integrals. Since such integrals necessarely enter the distribution function, the task of writing a global d.f. that accounts for the statistics of the total

Send offprint requests to: C. Efthymiopoulos, e-mail: cefthim@cc.uoa.gr of different families of orbits in a triaxial system seems hardly tractable.

Thus we are led to consider an old question of galactic dynamics, that of constructing "heuristic" models for the distribution function of collisionless stellar systems. Such models must be able to reproduce at least the "observables" i.e. mass and kinematic profiles of the real systems. At the same time, it is desirable that they are the least arbitrary possible, i.e. that they incorporate as much of our knowledge of the dynamical behaviour of stellar systems as possible.

In this paper we attempt to construct such a heuristic spherical model to approximate the coarse-grained distribution function of triaxial $N$-body systems. The systems studied here resulted from $N$-body experiments of "violent relaxation" from cold initial conditions (clumpy and non-clumpy) imitating the collapse of a protogalaxy in an expanding universe.

The method used here is a generalisation of an earlier work of one of us (Voglis 1994). The models of the following sections were derived using fitting procedures to the experimental data. However, we were able to "justify" them partially by analysing the statistics of individual stellar orbits. 
The numerical analysis benefited much from the use of a "smooth potential" code (Allen et al. 1990) for the $N$ body runs. In another paper (Contopoulos et al. 2001) we show that a smooth potential code can be used to calculate explicitely third integrals of motion for both the resonant and non-resonant orbits of the real $N$-body system. Thus we wish to stress the usefulness of the smooth potential codes in studying key questions concerning galactic 3D systems.

\section{The fitting method}

The orbits in a triaxial system in steady state are described by a $3 \mathrm{D}$ Hamiltonian of the form:

$H \equiv-\mathcal{E}=\frac{p^{2}}{2}-\Psi_{0}(r)-\Psi_{1}(r, \theta, \phi)$

where $\Psi_{0}(r)$ is minus the monopole (spherical) term of the potential and $\Psi_{1}(r, \theta, \phi)$ is a triaxial perturbation term of the potential.

If the term $\Psi_{1}$ is omitted, then the Hamiltonian (1) becomes the Hamiltonian of a spherical system. In that case, as it is well known, we can create a full and strict partition of phase space in terms of the invariant 2-tori of constant energy $\mathcal{E}$, and angular momentum $L^{2}$.

Let $\mathrm{d} \omega\left(\mathcal{E}, L^{2}\right)$ be the infinitesimal volume of phase space corresponding to the neighboring pairs of values $\left(\mathcal{E}, L^{2}\right)$ and $\left(\mathcal{E}+\mathrm{d} \mathcal{E}, L^{2}+\mathrm{d} L^{2}\right)$. According to the well known Jeans theorem, the distribution function $f$ depends on the phase space variables through the integrals of motion. In the case of spherical anisotropic systems we have $f \equiv f\left(\mathcal{E}, L^{2}\right)$. As a consequence, $f$ must have constant value within the whole infinitesimal volume $\mathrm{d} \omega$. This constant value is given by:

$f=\frac{\mathrm{d} N\left(\mathcal{E}, L^{2}\right)}{\mathrm{d} \omega\left(\mathcal{E}, L^{2}\right)}$

where $\mathrm{d} N\left(\mathcal{E}, L^{2}\right)$ is the number of particles with energies between $\mathcal{E}, \mathcal{E}+\mathrm{d} \mathcal{E}$ and angular momenta between $L^{2}$ and $L^{2}+\mathrm{d} L^{2}$. Equation (2) takes the form

$f=\frac{\mathrm{d} N\left(\mathcal{E}, L^{2}\right)}{\mathrm{d} \omega\left(\mathcal{E}, L^{2}\right)}=\frac{\frac{\mathrm{d} N\left(\mathcal{E}, L^{2}\right)}{\mathrm{d} \mathcal{E} \mathrm{d} L^{2}}}{\frac{\mathrm{d} \omega\left(\mathcal{E}, L^{2}\right)}{\mathrm{d} \mathcal{E} \mathrm{d} L^{2}}}=\frac{N\left(\mathcal{E}, L^{2}\right)}{\omega\left(\mathcal{E}, L^{2}\right)}$

where $N\left(\mathcal{E}, L^{2}\right)$ is the number density function, namely the number of particles per unit $\mathcal{E}$ and $L^{2}$, and $\omega\left(\mathcal{E}, L^{2}\right)$ is the density of states function, i.e. volume of phase space coresponding to a particular pair of values $\left(\mathcal{E}, L^{2}\right)$ per unit $\mathcal{E}$ and $L^{2}$. The latter function has the dimensions of time. It can be easily proved (e.g., Ogorodnikov 1965) that

$\omega\left(\mathcal{E}, L^{2}\right)=4 \pi^{2} T_{\mathrm{r}}\left(\mathcal{E}, L^{2}\right)$

where

$T_{\mathrm{r}}\left(\mathcal{E}, L^{2}\right)=2 \int_{r_{\mathrm{p}}}^{r_{\mathrm{a}}} \frac{\mathrm{d} r}{\sqrt{2\left[\Psi_{0}(r)-\mathcal{E}\right]-L^{2} / r^{2}}}$ is the radial period, i.e. the time needed to go from the pericenter $r_{\mathrm{p}}\left(\mathcal{E}, L^{2}\right)$ to the apocenter $r_{\mathrm{a}}\left(\mathcal{E}, L^{2}\right)$ and back to pericenter.

The functions $T_{r}\left(\mathcal{E}, L^{2}\right)$, and $\omega\left(\mathcal{E}, L^{2}\right)$ can be evaluated through the integration of Eq. (5). Thus, in order to find the distribution function $f\left(\mathcal{E}, L^{2}\right)$ we only need to find the number density function $N\left(\mathcal{E}, L^{2}\right)$. The latter is easily evaluated in $N$-body simulations by counting the number of particles per unit $\mathcal{E}$ and $L^{2}$ intervals in a grid on the plane $\left(\mathcal{E}, L^{2}\right)$.

If we consider now the full triaxial self-consistent system, we no longer have the integrals of motion of the spherical system. However, we can hope that some memory of the phase space structure of a spherical anisotropic system is still retained. In order to check this, we make the following construction: we keep the partition of phase space in terms of the subvolumes $\mathrm{d} \omega\left(\mathcal{E}, L^{2}\right)$, as in the case of spherical anisotropic systems, and we find the mean value of the distribution function in each such subvolume. Namely, the coarse grained distribution function of the real system is approximated by

$F=\frac{\mathrm{d} N\left(\mathcal{E}, L^{2}\right)}{\mathrm{d} \omega\left(\mathcal{E}, L^{2}\right)}=\frac{N\left(\mathcal{E}, L^{2}\right)}{\omega\left(\mathcal{E}, L^{2}\right)}$.

Here $\omega\left(\mathcal{E}, L^{2}\right)$ is calculated as in Eqs. (4) and (5) where $\Psi_{0}(r)$ is the monopole term of the potential expansion $\Psi_{0}(r)+\Psi_{1}(r, \theta, \phi)$. As we will see in Sect. 4, the numerical data show that both $N\left(\mathcal{E}, L^{2}\right)$ and $F$ remain invariant with respect to time when a system is at equilibrium, despite the fluctuations of the energy $\mathcal{E}$, and of the angular momentum $L^{2}$ of the particles' orbits. This is nothing more than a property of statistical equilibrium. We can exploit this property and obtain a fit for the function $F$ by fitting separately the invariant functions $N\left(\mathcal{E}, L^{2}\right)$ and $\omega\left(\mathcal{E}, L^{2}\right)$

We found that the coarse-grained distribution function $F$ evaluated in the above way gave average radial profiles of the density and of the radial anisotropy parameter for each system that were in excellent agreement with the corresponding experimental profiles. However, we do not expect that information related to the triaxiality of the system, such as the form of the local velocity ellipsoids, can be extracted from this function $F$. Such information can be found only if one knew a more complete form of the distribution function $f$ depending on three integrals of motion.

\section{3. $N$-body experiments: Initial conditions and numerical runs}

Two sets of initial conditions were used in the presented $N$-body experiments. These are "clumpy" and "nonclumpy" initial conditions. Both sets imitate the collapse of a protogalaxy in an Einstein-de Sitter expanding universe with density perturbations of a power law spectrum $P(k) \propto k^{n}$. Values of $n$ in the range $-3<n \leq 1$ represent the logarithmic slope of the power spectrum for different 
mass scales in the CDM scenario, where structures are formed by hierarchical clustering.

For all the simulations, the particles are placed initially in "quiet start" positions, namely at the nodes of a cubic grid within a sphere that has unit radius at decoupling. The particles are given velocities proportional to their distances from the center of the sphere (Hubble flow). The coefficient of proportionality (Hubble constant) is given the value required for the whole system to have zero total (kinetic+potential) energy. This ensures that the system expands in time as $R \propto t^{2 / 3}$ i.e. as in an Einstein-de Sitter universe.

"Non-clumpy" initial conditions are produced by perturbing the sphere with a spherical density perturbation which has the form of the rms profile of linear density perturbations corresponding to the power spectrum $P(k) \propto k^{n}$, namely the profile given by:

$\mu(h)=\left(<\left(\frac{\delta M}{M}\right)^{2}>\right)^{1 / 2}(h)=\frac{\mu_{0}}{h^{\frac{n+3}{2}}}$

where $h$ is the distance from the center of the sphere at decoupling. For a given radial profile of the form (7), the positions and velocities of the particles are perturbed according to the formulas (e.g., Palmer \& Voglis 1983):

$r=h \frac{1+\mu(h)}{2 \mu(h)}(1-\cos u)$

$\dot{r}=\frac{2 h \sqrt{\mu(h)}}{3 t_{0}} \frac{\sin u}{1-\cos u}$

where $u$ is the solution of

$t=t_{0} \frac{3[1+\mu(h)]}{4 \mu(h)^{3 / 2}}(u-\sin u)$

for a given time $t$. The time $t_{0}$ corresponds to the moment of decoupling.

These formulas are exact as long as there is no shell crossing (Palmer \& Voglis 1983), and they agree, to first order, with the Zel'dovich approximation (1970) for this particular perturbation field.

According to Eq. (7), $\mu_{0}$ is the amplitude of the perturbation at the unit scale $h=1$ at decoupling. By adjusting the value of $\mu_{0}$ we can control the collapse time of the sphere. The collapse time is given by $t_{\text {col }} \approx 3 t_{0} \pi / 2 \mu_{0}^{3 / 2}$. We take $t \simeq 1000 t_{0}$, corresponding to an average time when perturbations of the galactic mass scale become nonlinear. Thus we find $\mu_{0} \approx 0.025$. In fact we fix $\mu_{0}$ in such a way that the total energy of the perturbed sphere is the same for all the experiments, $E=-0.014$ in our units.

We present five experiments with non clumpy initial conditions, namely for the values $n \rightarrow-3$ and $n=$ $-2,-1,0,1$ of the power exponent. With $n \rightarrow-3$ we mean that we actually take a value of $n$ close to -3 , namely $n=-2.8$. The exact value $n=-3$ would lead to an exactly homogeneous spherical perturbation. At this limit the sphere would collapse theoretically to a point at the center, but the collapse is very unstable to small deviations from the radial flow. This of course is a limit which cannot be treated by any $N$-body simulation.

As regards "clumpy" initial conditions, we apply the usual procedure of perturbing the sphere with the formulas of Zel'dovich approximation, considering perturbations of all scales within the sphere. The perturbation field $A(\boldsymbol{r})$ is defined by its Fourier transform. If $\boldsymbol{q}$ is the unperturbed position of a particle at decoupling, then the density perturbation field at this position has the value

$$
A(\boldsymbol{q})=A_{0} \sum \sum_{k_{\min }}^{k_{\max }} \sum \sqrt{P(k)} \cos \left(k_{x} q_{x}+k_{y} q_{y}+k_{z} q_{z}+\phi_{k}\right)
$$

where $P(k)=k^{n}$ is the power spectrum, and we add waves of wavenumbers between two limits $k_{\min }<k<k_{\max }$ with random phases $\phi_{k}$. We take $k_{\max }$ equal to the Nyquist wavenumber of the grid, which corresponds to the resolution wavelength. Then we add a discrete number of waves in a grid in the wavenumber space with step $k_{\min }=$ $2 \pi / 4 R$, where $R$ is the radius of the system. This means that the contribution of wavelengths longer than $4 R$ is ignored in Eq. (11). This contribution is "seen" almost as a uniform background within the scale of the sphere, because the oscillations of the corresponding waves are on much larger scales. In order to add this uniform background we take the overall perturbation equal to:

$A^{\prime}(\boldsymbol{q})=A(\boldsymbol{q})+A_{\mathrm{c}}$

where $A_{\mathrm{c}}$ is calculated as the expected rms value of the perturbation at the scale $R$.

The sequence of phases $\phi_{k}$ are produced by a random number generator. We try many different sequences $\phi_{k}$ and choose one that creates a substantial overdensity in the scale of the sphere. The criterion for this is the following. We perturb the positions and velocities of particles according to the Zel'dovich approximation:

$$
\begin{aligned}
& \boldsymbol{r}_{\boldsymbol{i}}=a(t)\left[\boldsymbol{q}_{i}-b(t) \boldsymbol{\Psi}\left(\boldsymbol{q}_{i}\right)\right] \\
& \boldsymbol{v}_{\boldsymbol{i}}=a(t)\left[H(t) \boldsymbol{q}_{i}-H(t) b(t) \boldsymbol{\Psi}\left(\boldsymbol{q}_{i}\right)-\dot{b}(t) \boldsymbol{\Psi}\left(\boldsymbol{q}_{i}\right)\right]
\end{aligned}
$$

where $\boldsymbol{\Psi}(\boldsymbol{q})$ is defined as

$$
\begin{aligned}
\boldsymbol{\Psi}(\boldsymbol{q})= & A_{0} \sum \sum_{k_{\min }}^{k_{\max }} \sum \sqrt{P(k)} \frac{\boldsymbol{k}}{k^{2}} \\
& \times \sin \left(k_{x} q_{x}+k_{y} q_{y}+k_{z} q_{z}+\phi_{k}\right),
\end{aligned}
$$

$a(t)=\left(\frac{t}{t_{0}}\right)^{2 / 3}$ is the scale factor, $H(t)=\dot{a} / a$ is the Hubble parameter, $b(t)=t^{2 / 3}$, and $A_{0}$ is the normalization constant, i.e. the amplitude of the spectrum. The criterion that a system has formed a global overdensity is the sign of the total energy of the perturbed sphere, which should be negative. The total energy is the sum of many terms which may be positive or negative. Thus essentially the sign of the total energy depends on the sequence $\phi_{k}$. For a fixed sequence $\phi_{k}$, the absolute value of the energy depends on the amplitude $A_{0}$ of the perturbations. For each 
experiment, we set $A_{0}$ in such a way so that the total energy is $E=-0.014$, the selected constant value of the energy for all the experiments.

We present four experiments with clumpy initial conditions, for the values $n=-3,-2.5,-2,-1$ of the power spectrum exponent.

The above initial conditions represent simple models of collapse in an expanding universe where structures form by hierarchical clustering. However, they contain all the essential features that are needed to produce $N$-body systems in stellar dynamical equilibrium formed by collapse and violent relaxation. In particular, the "non-clumpy" experiments are subject to the well known radial orbit instability that leads to triaxial systems with axial ratios $\approx 0.5$. On the other hand, the "clumpy" experiments lead to objects with moderate triaxiality. In both cases the value of the exponent $n$ of the power spectrum determines, through Eq. (10) the timescale at which a clump of scale length $h$ at decoupling collapses. Thus for $n=-3$, $\mu(h)=$ const., i.e. the same for all scale lengths. In this case, the perturbations of all scale lengths collapse simultaneously. This is the most violent possible collapse. On the other hand, the collapse is less violent as $n$ increases above the value -3 . Thus, by changing $n$ we control the violence of the relaxation for the different experiments.

\subsection{Numerical runs}

The numerical technique used for the simulations is the same as that in Voglis (1994). Briefly, we use the tree code of Hernquist (1987) to simulate the collapse phase of each $N$-body system. This phase lasts for only a few dynamical periods, and it is followed by a much slower phase of approach to steady state equilibrium. The latter phase is simulated by a different code, the smooth field code (conservative technique code) of Allen et al. (1990). The advantages of using this combination of codes is explained in detail in Voglis (1994).

In Fig. 1 we show some snapshots of the collapse phase for some of our experiments. We refer to each of the experiments with non-clumpy initial conditions as "experiment Sn", where $\mathrm{n}$ takes the values $-3,-2,-1,0$ and 1 (as explained in Sect. 3, $n=-2.8$ for the experiment S-3). The experiments with clumpy initial conditions are called "Cn", where $n=-3,-2.5,-2$ and -1 . In Fig. 1 we have the time evolution for the experiments S-3 (Fig. 1a), S-1 (Fig. 1b), C-3 (Fig. 1c) and C-1 (Fig. 1d).

The evolution of the non-clumpy and of the clumpy experiments is different. In the non-clumpy cases (Figs. 1a,b), the collapse starts forming one clump at the center which grows in size due to the prolonged secondary infall of matter. This structure is initially spherically symmetric, but later on it loses its spherical symmetry due to a "radial orbit instability". This kind of instability is well known (e.g., Polyachenko \& Shukman 1981; Fridman \& Polyachenko 1984; Barnes et al. 1986; Palmer \& Papaloizou 1987) to occur in systems dominated by radial orbits, as in all the Sn experiments. As a result, at the end of the collapse all the Sn systems are strongly flattened, with axial ratios corresponding to E5-E6 systems. These systems are triaxial in general.

In the clumpy cases (Figs. 1c,d) instead of one structure at the center we have several structures (clumps) formed at random places within the bound sphere. The clumps then merge to the center and form a final equilibrium system. In all the $\mathrm{Cn}$ experiments the final systems are also triaxial but with moderate flattening (E2-E3). This flattening is due rather to the initial conditions than to the radial orbit instability. In fact, the various clumps formed initially heat the system in the transverse direction of motion and prevent it from being subject to the radial orbit instability.

\section{Simple fitting model of the function $N\left(\mathcal{E}, L^{2}\right)$}

In Fig. 2 we plot the number density function $N\left(\mathcal{E}, L^{2}\right)$ as found directly from the $N$-body data for all the experiments at equilibrium. In these histograms the horizontal axis is the axis of the absolute energy $\mathcal{E}=-E$ (called simply "energy"). The energy $\mathcal{E}$ increases from right to left. The other axis of the horizontal plane is the axis of the angular momentum $L^{2}$. The $z$-axis gives the value of $N\left(\mathcal{E}, L^{2}\right)$.

The distributions $N\left(\mathcal{E}, L^{2}\right)$ remain invariant in time for all the experiments. This is shown in Fig. 3, where the contours of $N\left(\mathcal{E}, L^{2}\right)$ on the $\left(\mathcal{E}, L^{2}\right)$ plane are plotted for the experiment S-2 and for two different snapshots differing by one hundred dynamical periods, namely $t=$ 100 (solid) and $t=200$ (dashed). The sets of contours almost coincide for the two time snapshots.

The invariance of $N\left(\mathcal{E}, L^{2}\right)$ is not trivial, since neither $\mathcal{E}$ nor $L^{2}$ are conserved along the individual stellar orbits. The energy $\mathcal{E}$ is subject to a small variation due to the noise of the smooth potential code that causes a small time variation of the coefficients of the potential expansion. On the other hand, the angular momentum $L^{2}$ of an orbit is subject to large variations due to the fact that the system is triaxial. Despite these variations of $\mathcal{E}$ and $L^{2}$ for each individual orbit, we found that the distribution $N\left(\mathcal{E}, L^{2}\right)$ remains invariant due to statistical equilibrium. Namely, a given cell $(\mathcal{E}, \mathcal{E}+\Delta \mathcal{E}),\left(L^{2}, L^{2}+\Delta L^{2}\right)$ does not contain the same particles for all times. However, the cells exchange particles in such a way that the number of particles in each cell remains practically constant.

There are three main characteristics observed in Figs. 2a to 2i:

1. A region where $N\left(\mathcal{E}, L^{2}\right)=0$, i.e. occupied by no particles. This region is limited by a virtual curve in each panel of Fig. 2. This curve is close to the curve giving the energy $\mathcal{E}_{\mathrm{c}}\left(L^{2}\right)$ for which the motion under the influence of the monopole potential term $\Psi_{0}(r)$ would be a circular orbit with angular momentum $L^{2}$, namely:

$\mathcal{E}_{\mathrm{c}}\left(L^{2}\right)=\Psi_{\mathrm{o}}\left(r_{\mathrm{c}}\right)-\frac{L^{2}}{2 r_{\mathrm{c}}^{2}}$, 

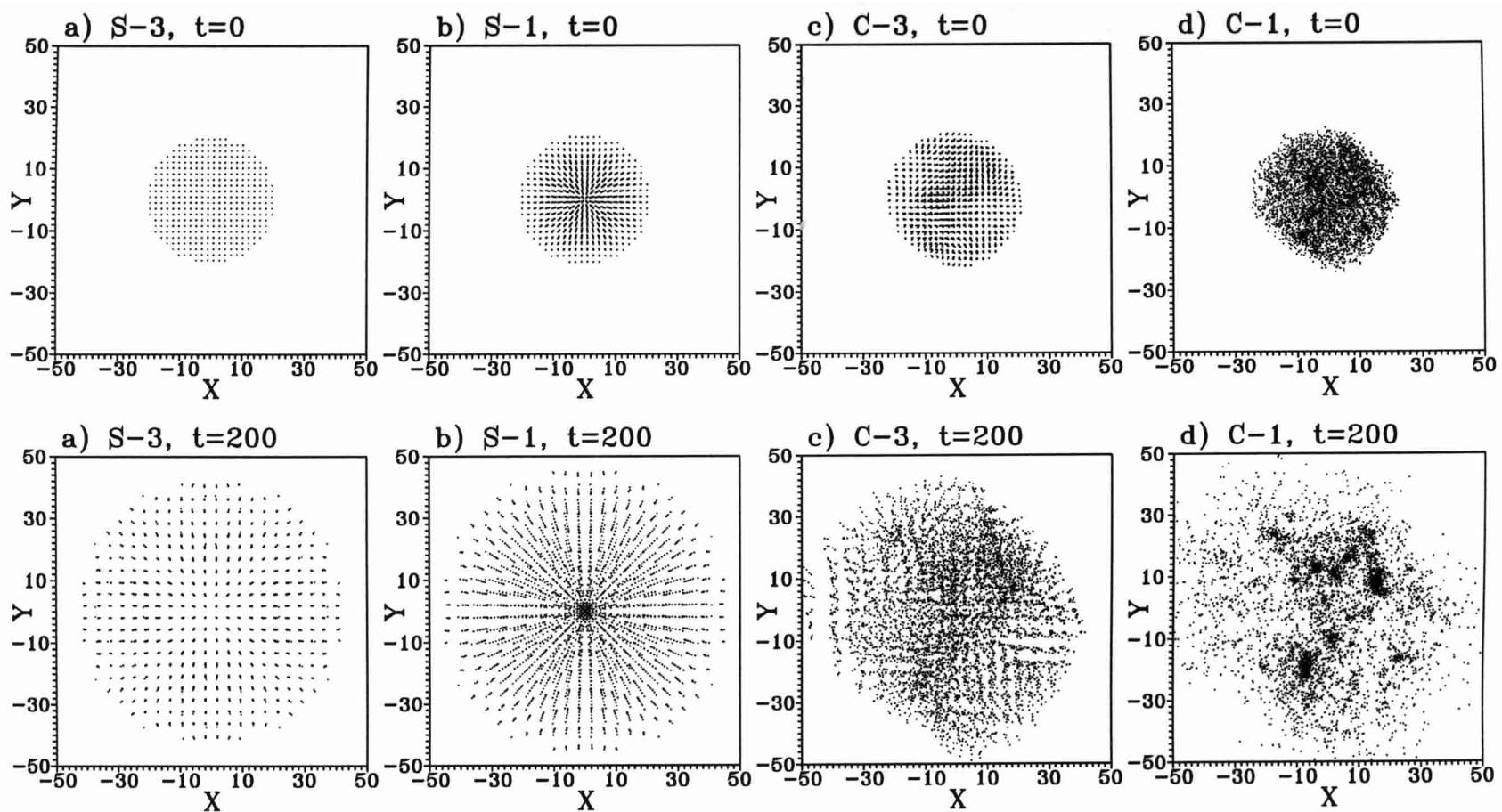

b) $\mathrm{S}-1, \mathrm{t}=200$

c) $\mathrm{C}-3, \mathrm{t}=200$

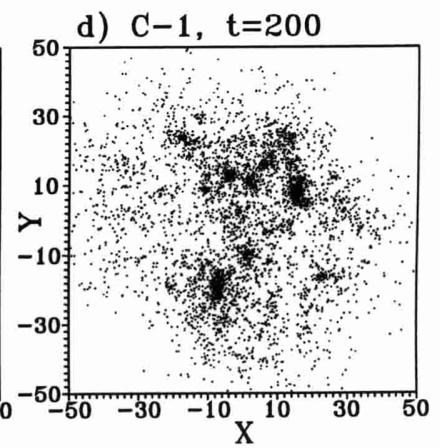

a) $\mathrm{s}-3, \mathrm{t}=400$

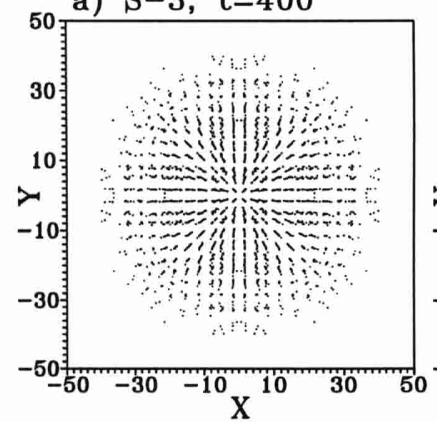

b) $\mathrm{S}-1, \mathrm{t}=400$

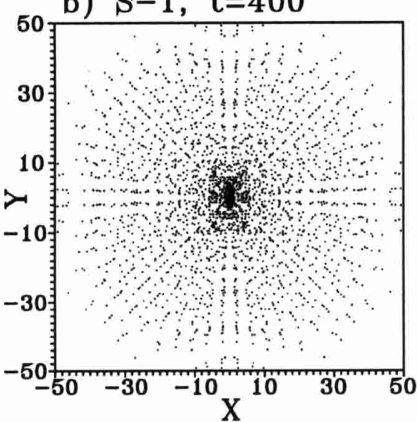

c) $\mathrm{C}-3, \mathrm{t}=400$

d) $\mathrm{C}-1, \mathrm{t}=400$

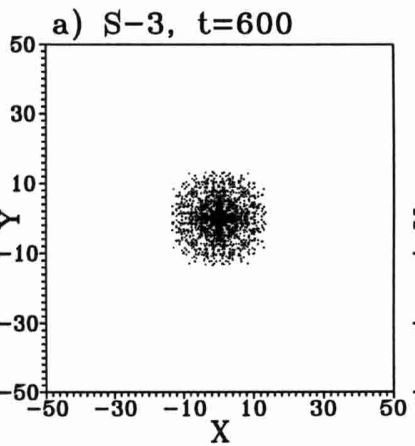

b) $s-1, t=600$

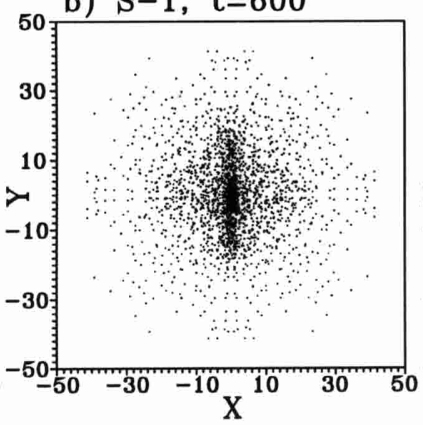

c) $\mathrm{C}-3, \mathrm{t}=600$

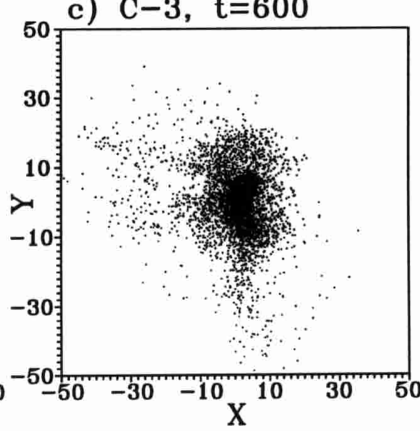

d) $\mathrm{C}-1, \mathrm{t}=600$

a) $\mathrm{s}-3, \mathrm{t}=1000$

b) $\mathrm{s}-1, \mathrm{t}=\mathbf{1 0 0 0}$
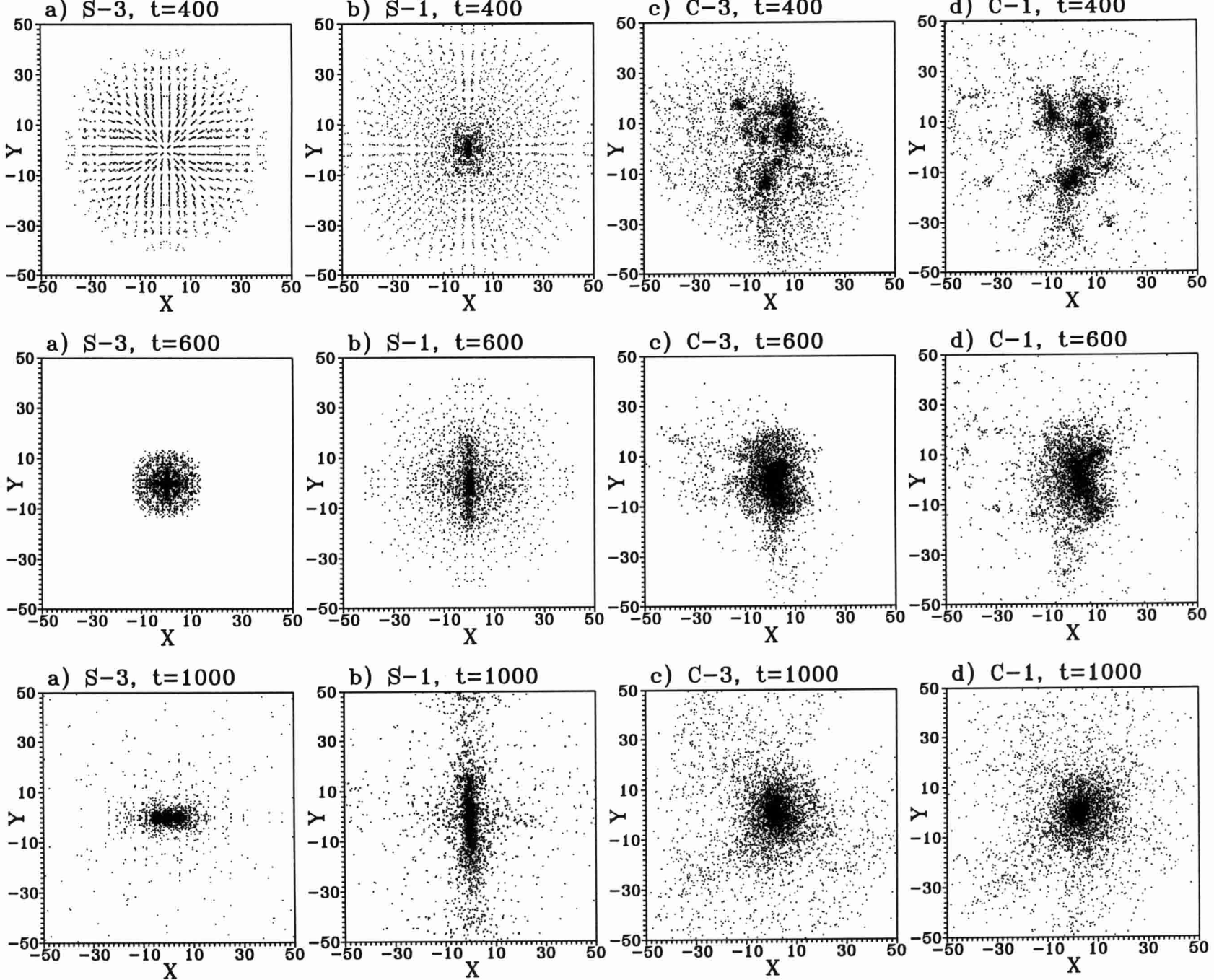

Fig. 1. Projection on the $x y$ plane of several snapshots of the time evolution of the experiments a) S-3 (first column), b) S-1 (second column), c) C-3 (third column), and d) C-1 (fourth column). 


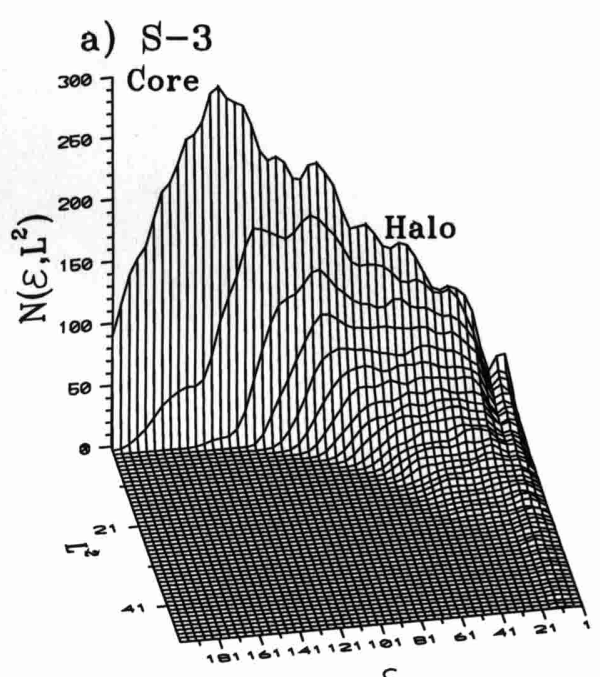

$\varepsilon$

d) $\mathrm{SO}$
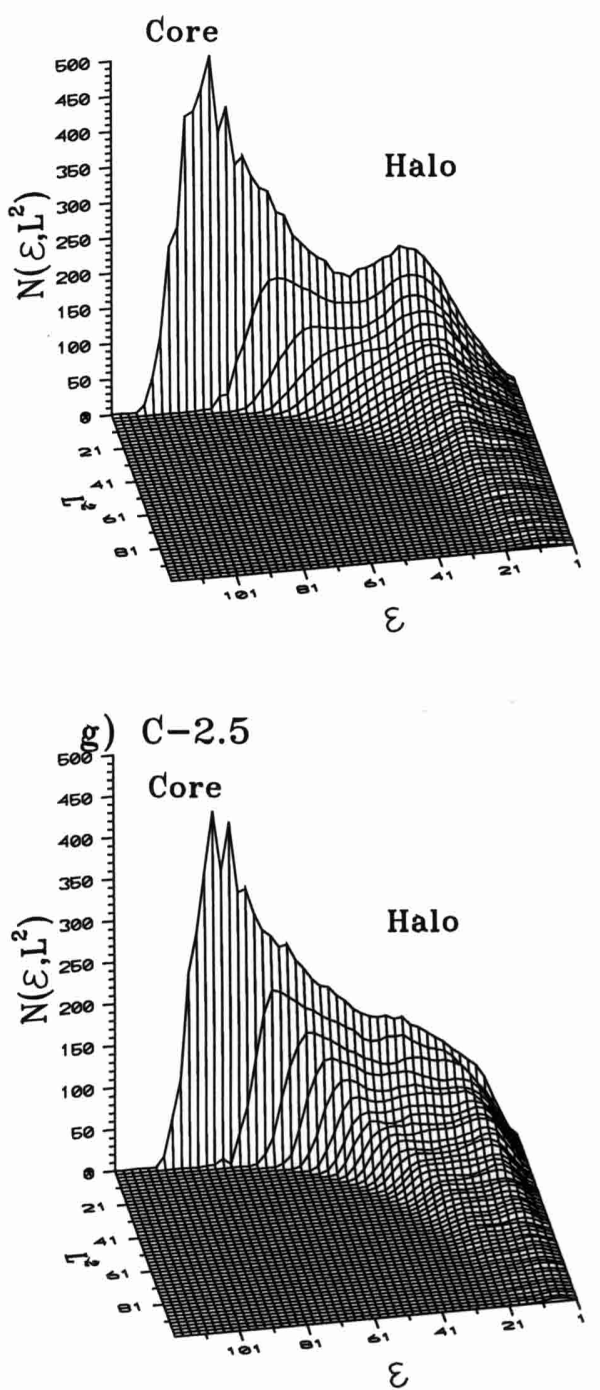

b) $\mathrm{s}-2$

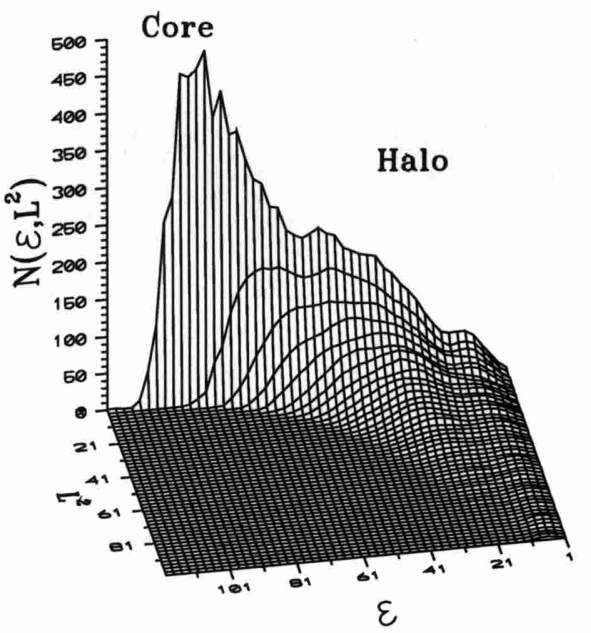

e) $\mathrm{S} 1$
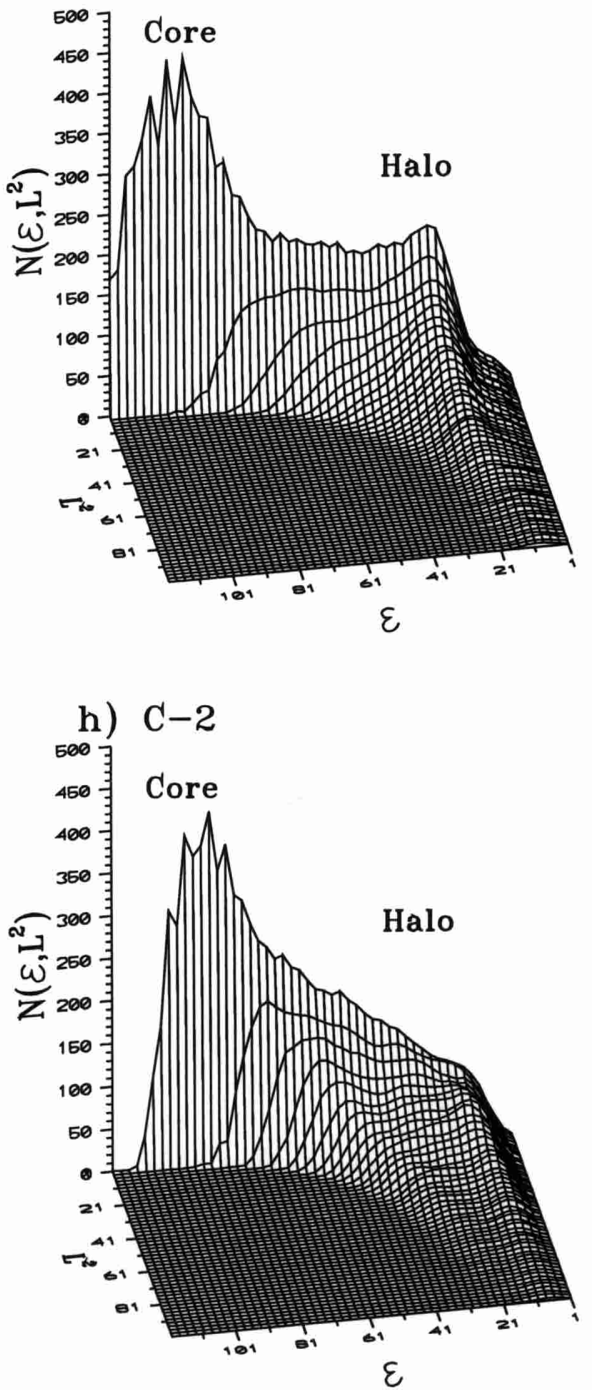

c) $\mathrm{S}-1$

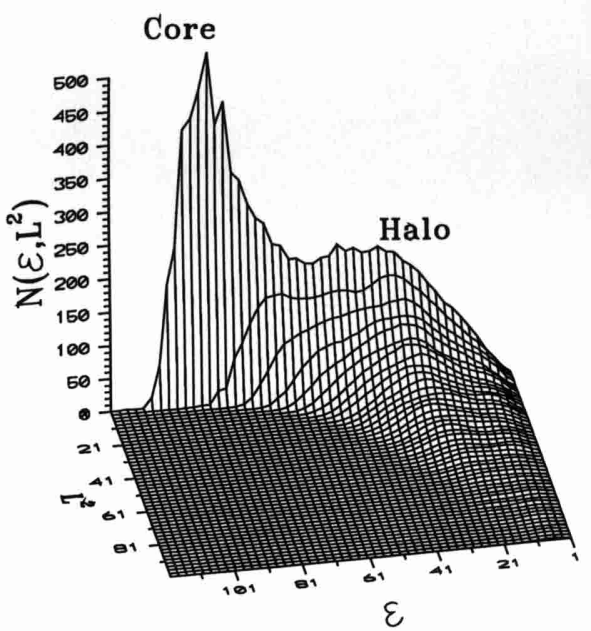

f) $\mathrm{C}-3$

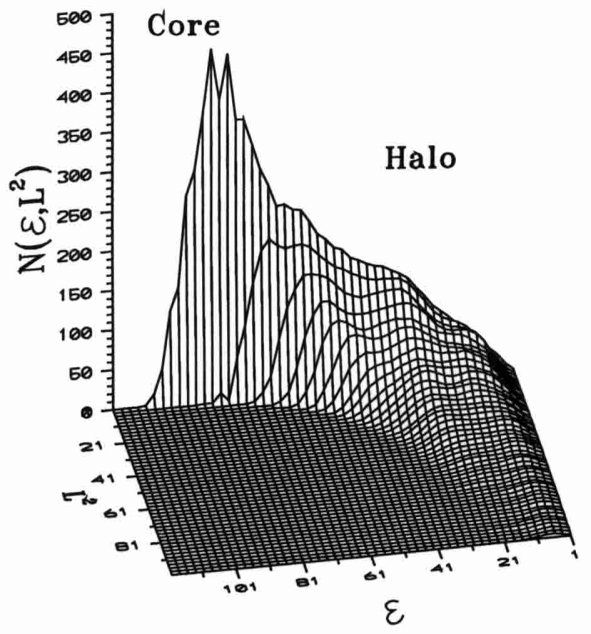

i) $\mathrm{C}-1$

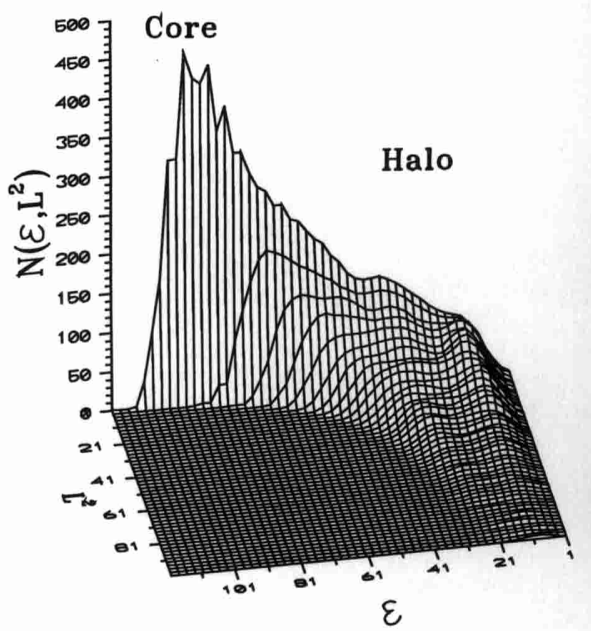

Fig. 2. Numerical histograms of the number density function $N\left(\mathcal{E}, L^{2}\right)$ for all the experiments. 


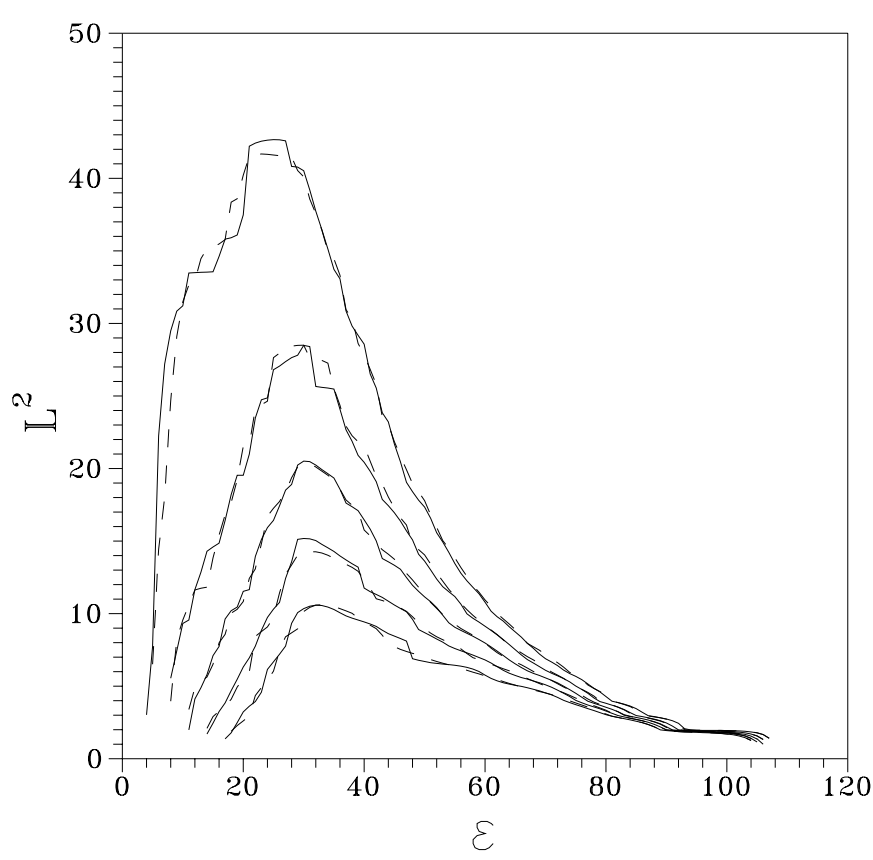

Fig. 3. The contours of $N\left(\mathcal{E}, L^{2}\right)$ on the $\left(\mathcal{E}, L^{2}\right)$ plane for the experiment S-2 and for two different snapshots, $t=100$ (solid) and $t=200$ (dashed).

where $r_{\mathrm{c}}\left(L^{2}\right)$ is the root for $r$ of the equation:

$$
\frac{\mathrm{d} \Psi_{\mathrm{o}}(r)}{\mathrm{d} r}+\frac{L^{2}}{r^{3}}=0
$$

If only the monopole term $\Psi_{0}(r)$ was considered, the motion would not be allowed in the region $\mathcal{E}>\mathcal{E}_{\mathrm{c}}\left(L^{2}\right)$. As a consequence, $N\left(\mathcal{E}, L^{2}\right)$ should have a sharp cut-off beyond the curve $\mathcal{E}_{\mathrm{c}}\left(L^{2}\right)$. However, due to the influence of the multipole potential terms, the real cut-off is not sharp. $N\left(\mathcal{E}, L^{2}\right)$ falls rapidly, but smoothly to zero for values $\mathcal{E}$ exceeding $\mathcal{E}_{\mathrm{c}}\left(L^{2}\right)$ towards the left;

2. A prominent maximum of the function $N\left(\mathcal{E}, L^{2}\right)$ is observed in the region of large binding energies and small angular momenta. This maximum is noted in all the diagrams as the "core", because most particles around this maximum move on orbits close to the center of the system;

3. A second locus of maxima of the function $N\left(\mathcal{E}, L^{2}\right)$ is observed in the region of small binding energies, extending to much higher values of the angular momentum than the core maximum. This locus is also noted in all the experiments as the "halo", because the particles around it move on orbits reaching large distances from the center.

Voglis (1994) proposed an empirical model for fitting the function $N\left(\mathcal{E}, L^{2}\right)$. The model has the form:

$N\left(\mathcal{E}, L^{2}\right)=\frac{N_{\mathrm{h}}\left(\mathcal{E}, L^{2}\right)+N_{\mathrm{c}}\left(\mathcal{E}, L^{2}\right)}{\exp \left[\beta\left(\mathcal{E}-\mathcal{E}_{\mathrm{c}}\right]+1\right.}$

where $N_{\mathrm{c}}\left(\mathcal{E}, L^{2}\right)$ is a core term and $N_{\mathrm{h}}\left(\mathcal{E}, L^{2}\right)$ a halo term. The denominator of Eq. (18) provides a natural cut-off of the distribution $N\left(\mathcal{E}, L^{2}\right)$ at the energy of the circular orbit $\mathcal{E}_{\mathrm{c}}$.

The fitting model is completely defined by specifying the form of the functions $N_{\mathrm{c}}\left(\mathcal{E}, L^{2}\right)$ and $N_{\mathrm{h}}\left(\mathcal{E}, L^{2}\right)$. Voglis (1994) proposed a polytropic law $N_{\mathrm{c}}\left(\mathcal{E}, L^{2}\right)=N_{0} \mathcal{E}^{\mathrm{p}}$ for the core term. The halo term was fitted through empirical formulas (Voglis 1994) that fit the shape of the contours of $N\left(\mathcal{E}, L^{2}\right)$ in the halo region of the $\left(\mathcal{E}, L^{2}\right)$ plane. These formulas are based on the assumption that a contour has a simple shape with one local maximum of $\mathcal{E}$ with respect to $L^{2}$ for each fixed value of $N$. In Figs. $4 a-$ e we see that for the non-clumpy experiments, these local maxima are delineated along simple loci (dashed lines) on the $\left(\mathcal{E}, L^{2}\right)$ plane. For these experiments, the model of Voglis (1994) fits nicely the distribution $N\left(\mathcal{E}, L^{2}\right)$, as shown in Fig. 5, for an appropriate choice of the model's fitting parameters. However, the model does not work adequately for the experiments with "clumpy" initial conditions. In the contour diagrams of Figs. $4 \mathrm{f}-\mathrm{i}$ we see that the contours have more complicated shapes than those of the non-clumpy experiments (Figs. 4a-e). In particular, most contours have more than one "halo" maxima, and they cannot be fitted by simple formulae. This complicated structure of the halo region is expected since the systems relax from more complicated initial conditions, characterized by the existence of various clumps that collapse in different timescales. This fact was a motivation to look for a more general model for $N\left(\mathcal{E}, L^{2}\right)$. We found that such a model can be obtained if we decompose $N\left(\mathcal{E}, L^{2}\right)$ in terms of "spherical shell number density functions", as described in the next section.

Before discussing the new model it is worth making some comments on the similarities and the differences between our results and the results found by Aguilar \& Merritt (1990, Figs. 17 and 18). These authors do not find a prominent core maximum as in our experiments. We found that this is due to a smoothing effect. Namely, the bin size in the angular momentum necessary to make the core maximum visible is one order of magnitude smaller than the size used in Fig. 17 of Aguilar and Merritt. Such a small bin is allowed here because the number of particles in our simulations is large enough.

What is common in both analyses is the existence of a negative temperature area $\left(\beta=\frac{\partial N}{\partial \mathcal{E}}<0\right)$. However, our analysis shows that the area that can be fitted with a negative temperature model is limited due to two reasons: a) the existence of the core population, and b) the depopulation of particles in small binding energies $\mathcal{E}$. (See also the discussion in Sect. 6 of Voglis 1994.)

\section{The spherical shell number density function}

To demonstrate the new model, we use as an example the data of the experiment C-2. Let the system be divided in a number of successive spherical shells of infinitesimal width $\Delta r$. The "spherical shell number density function" is the function

$\nu\left(\mathcal{E}, L^{2}, r\right) \equiv \frac{\Delta N\left(\mathcal{E}, L^{2}, r\right)}{\Delta r \Delta \mathcal{E} \Delta L^{2}}$ 
a) $\mathrm{S}-3$

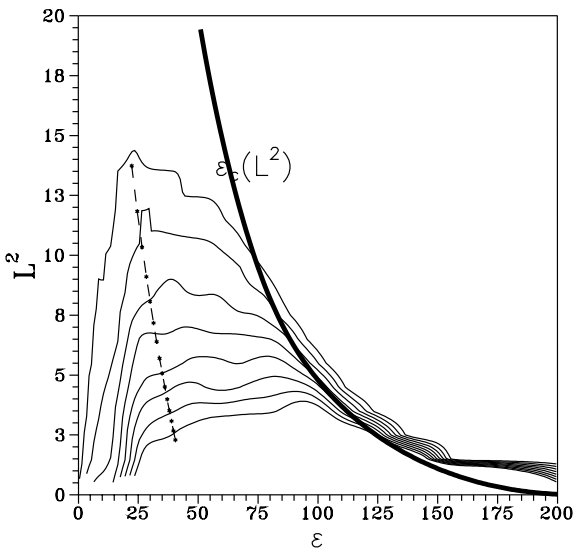

d) $\mathrm{SO}$

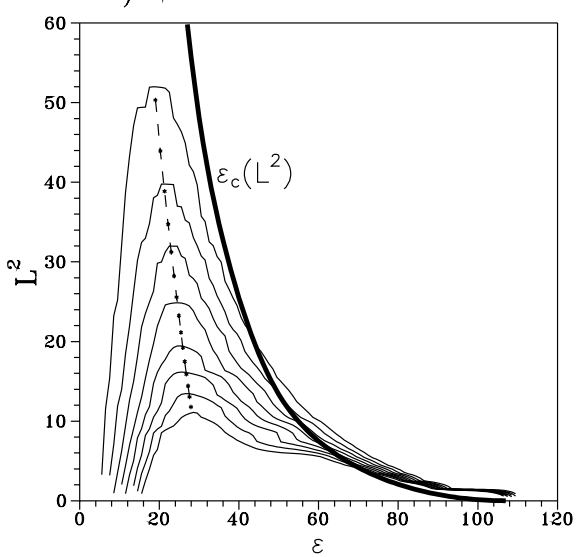

g) $\mathrm{C}-2.5$

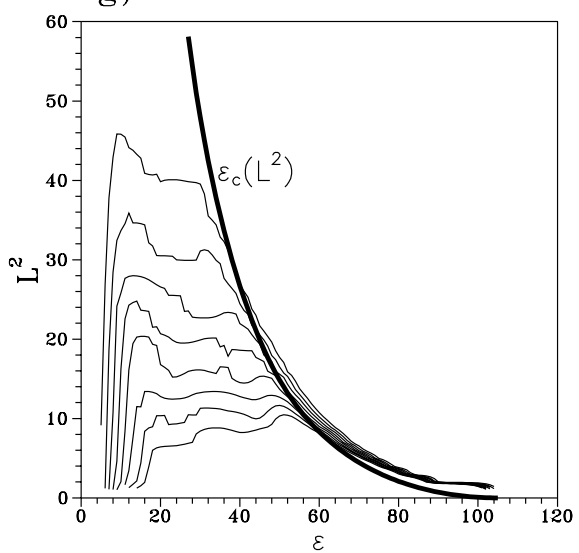

b) $S-2$

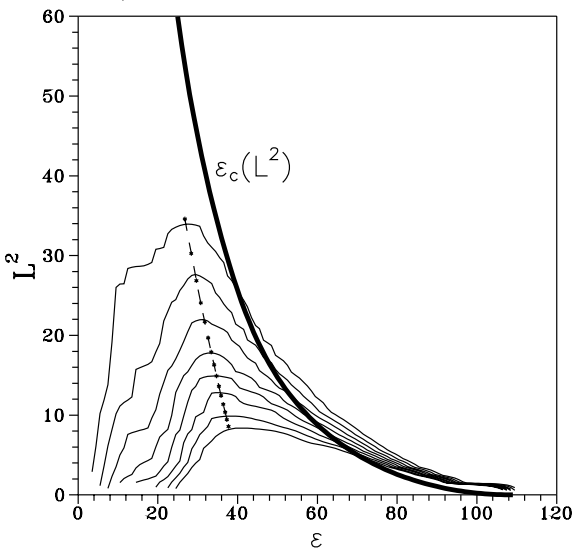

e) $\mathrm{S} 1$

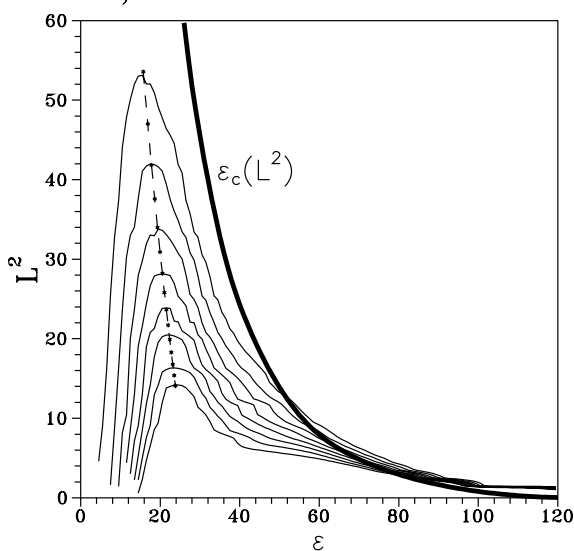

h) $\mathrm{C}-2$

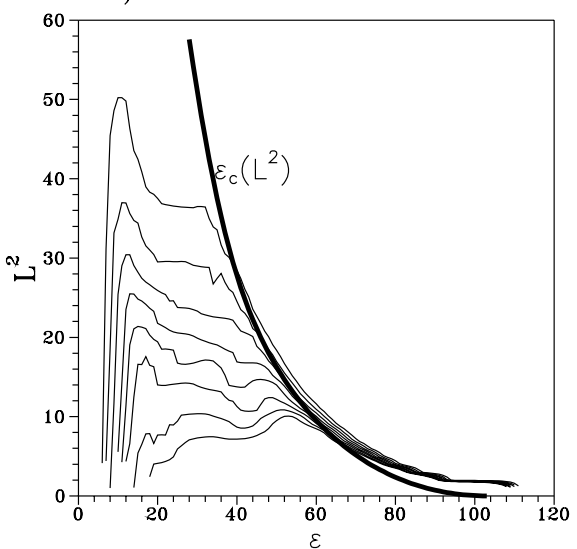

c) $\mathrm{S}-1$

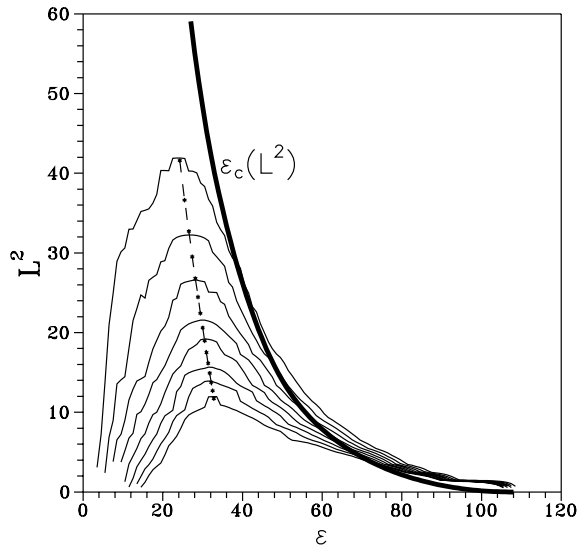

f) $\mathrm{C}-3$

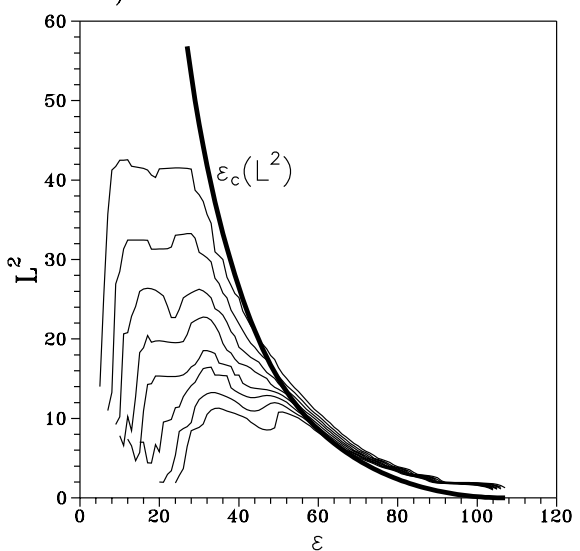

i) $\mathrm{C}-1$

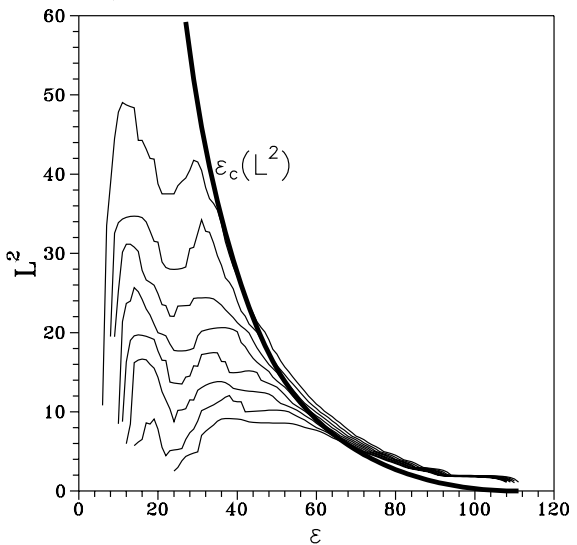

Fig. 4. Contour diagrams of the number density function $N\left(\mathcal{E}, L^{2}\right)$ on the plane $\left(\mathcal{E}, L^{2}\right)$ for all the experiments. The curve $\mathcal{E}_{\mathrm{c}}\left(L^{2}\right)$ of the circular orbit (solid) and the curve passing through the unique halo maximum of the non clumpy experiments a to e (dashed), are shown.

that gives the number of particles per unit radius, energy and angular momentum in a shell of radius $r$.

In Figs. 6a-d the contours of the function $\nu\left(\mathcal{E}, L^{2}, r\right)$ are shown for four different shells of radii $r=0.05,0.1$, 0.2 and 0.3 respectively. In all four panels, the contours of the function $\nu\left(\mathcal{E}, L^{2}, r\right)$ have a well defined maximum. The maxima of the contours of one panel are delineated along an almost perfectly straight line. The solid line with dots marked in Figs. 6a-d fits accurately the line of maxima of the contours. This line corresponds to orbits having their apsides in the shell and it is given by:

$L^{2}=-2 r^{2}\left[\mathcal{E}-\Psi_{\mathrm{o}}(r)\right]$,

or, solving for the energy

$\mathcal{E}=\Psi_{0}(r)-\frac{L^{2}}{2 r^{2}} \equiv \Psi_{\mathrm{e}}\left(r, L^{2}\right)$.

For constant values of $\mathcal{E}$ and $L^{2}$, Eq. (21) has two roots of $r$. These roots correspond to the distance of the pericenter 

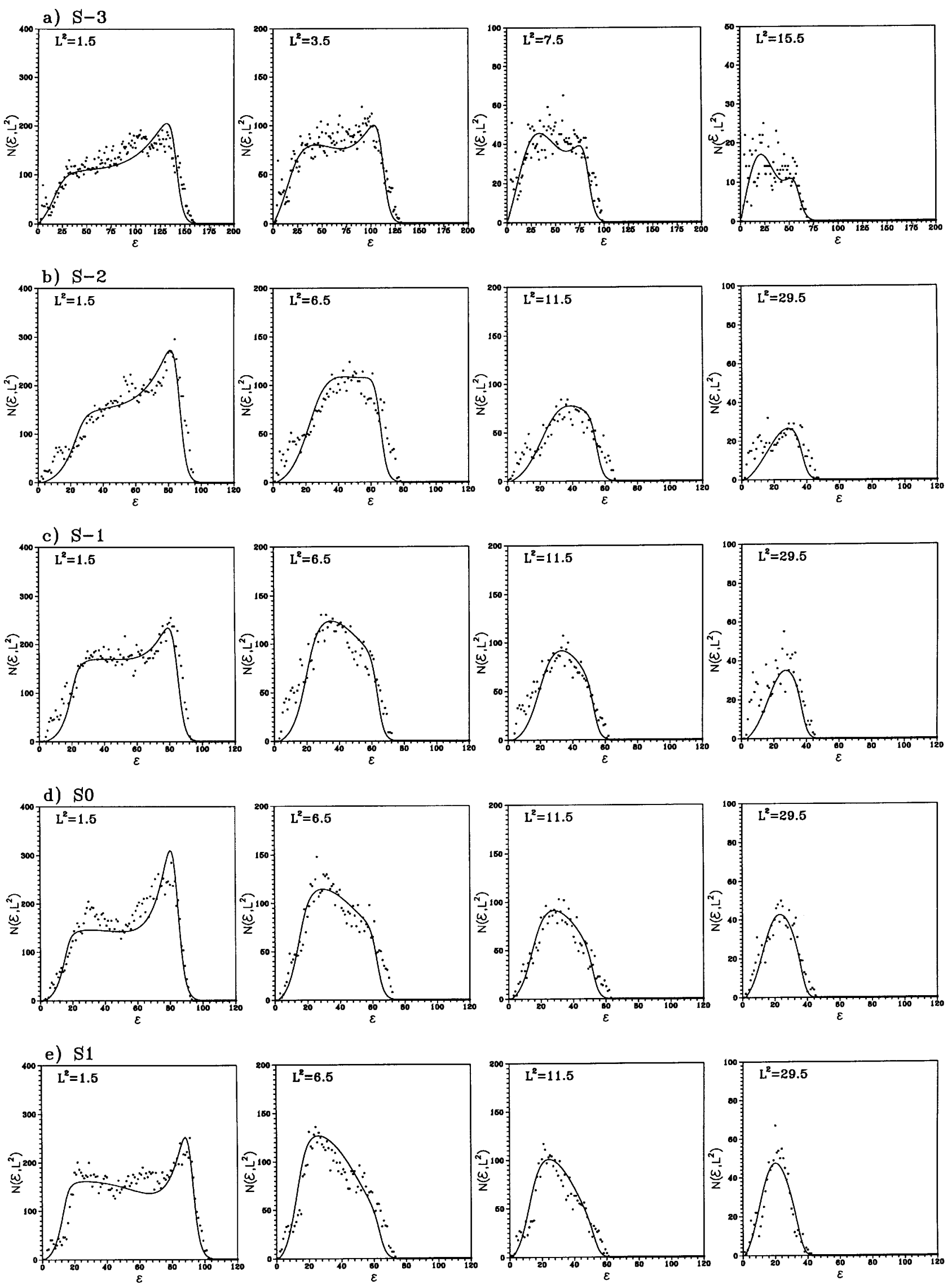

Fig. 5. The fitting of $N\left(\mathcal{E}, L^{2}\right)$ (dots) by the model of Eq. (18) (solid curve) for all the non clumpy (Sn) experiments. For each experiment, the fitting is shown along the energy axis, for four different slices of constant angular momentum $L^{2}$. 

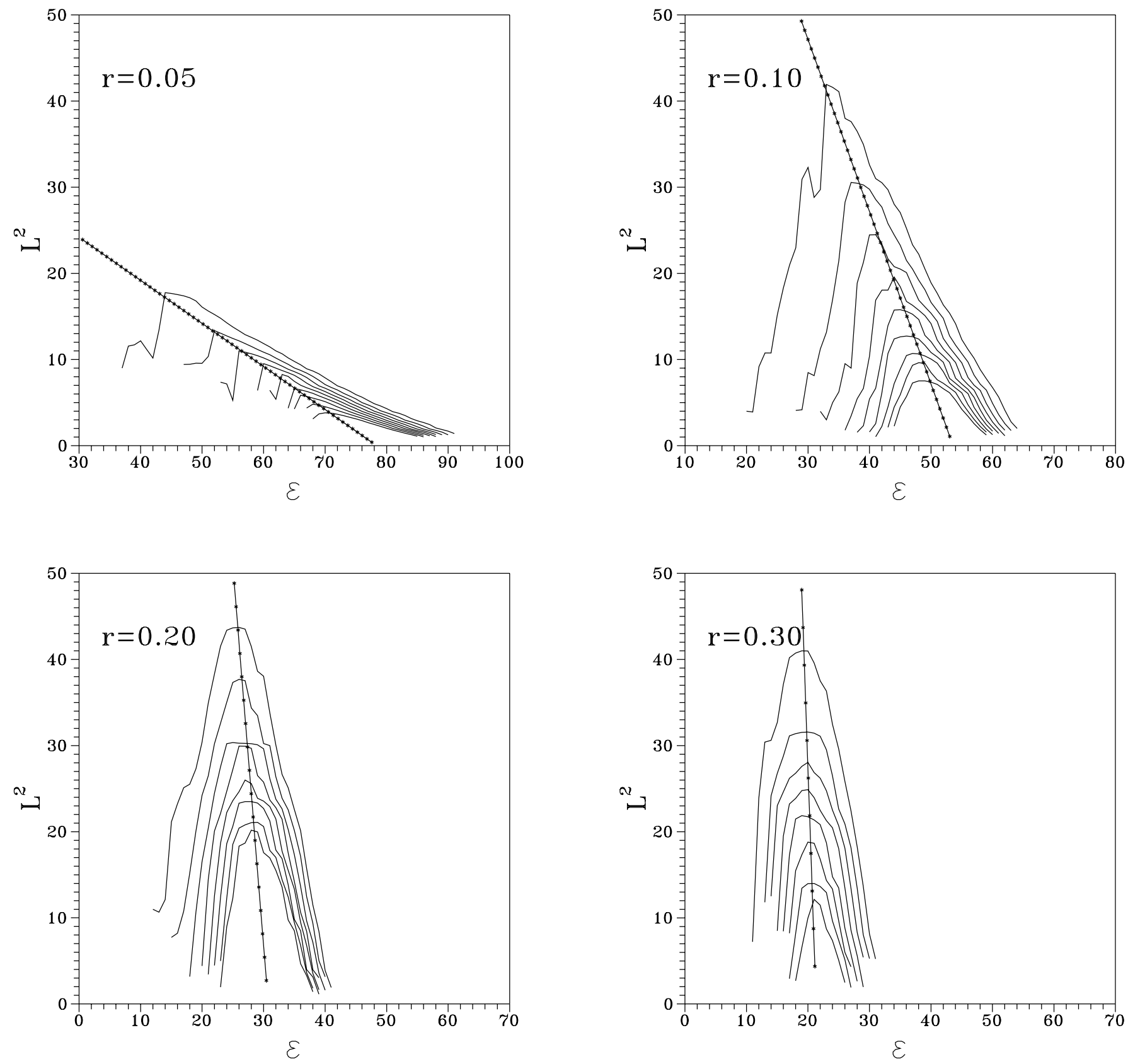

Fig. 6. Contours of the spherical shell number density function $\nu\left(\mathcal{E}, L^{2}, r\right)$ for the experiment C-2 and for the shells of radii a) $r=0.05$, b) $r=0.1$, c) $r=0.2$ and d) $r=0.3$. The dotted lines are given by Eq. (21).

and of the apocenter of the motion that a particle would do under the influence of the monopole potential term $\Psi_{0}(r)$. The fact that the distribution $\nu\left(\mathcal{E}, L^{2}, r\right)$ has maximum on the line given by Eq. (21) is understood by the following simple argument. Consider a particle entering the shell $(r, \Delta r)$ at some time $t$. The time spent by the particle within the shell is given by:

$\Delta t=\int_{r}^{r+\Delta r} \mathrm{~d} r / \dot{r}$

The probability that the particle stays within the shell is proportional to the time $\Delta t$. The particles for which $\dot{r}=0$ when they reach the cell spend their maximum time in the shell. For $\dot{r}=0$, the energy of a particle is given by:

$\mathcal{E}=-\frac{L^{2}}{2 r^{2}}+\Psi_{\mathrm{o}}(r)+\Psi_{1}(r, \theta, \phi)$

where $\Psi_{1}(r, \theta, \phi)$ is the sum of the multipole terms of the potential. If we average Eq. (23) over all the possible angles $\theta$ and $\phi$ we obtain Eq. (21).

We focus now on the form of the function $\nu\left(\mathcal{E}, L^{2}, r\right)$ for a fixed value of $r$. In Fig. 7a the function $\nu\left(\mathcal{E}, L^{2}, r\right)$ is plotted in logarithmic scale vs. the energy, for the shell $r=0.2$ and for the values of the angular momentum 
(a)

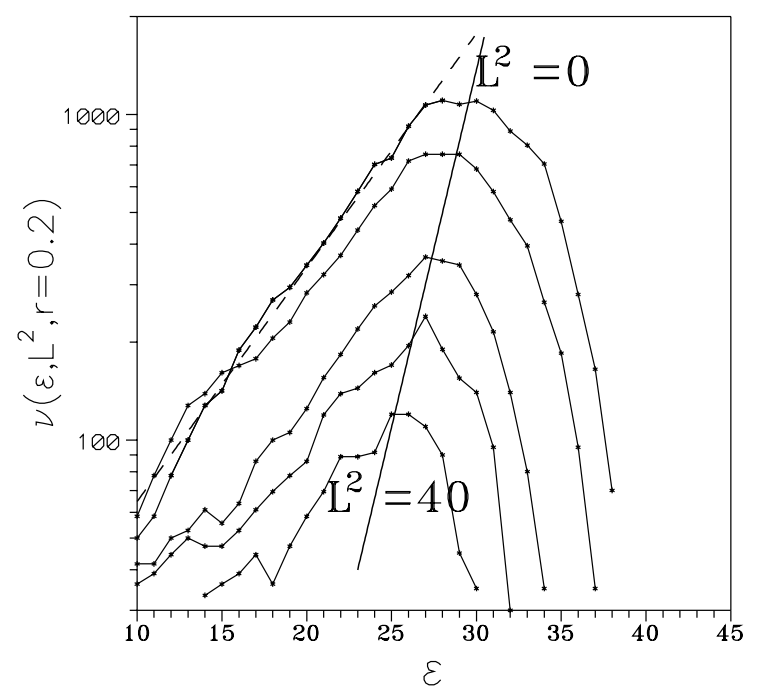

(b)

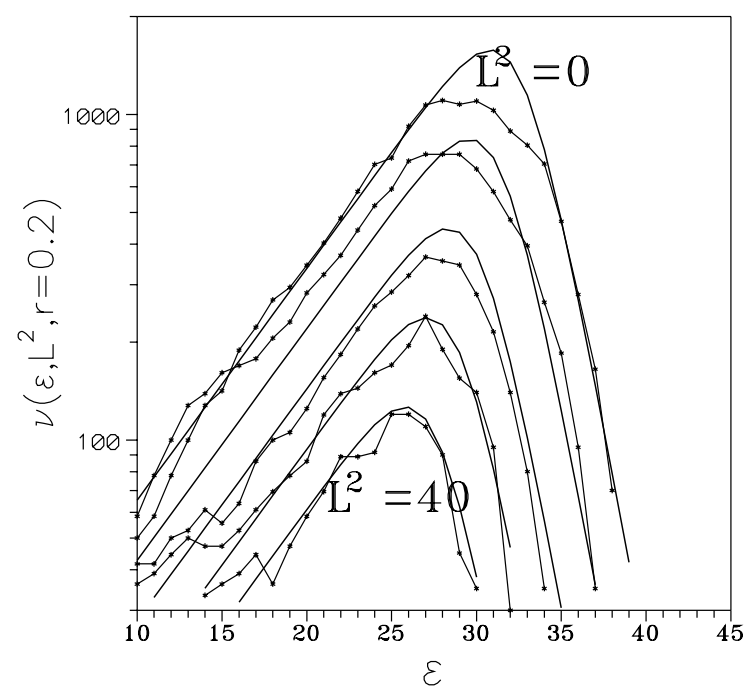

Fig. 7. a) The function $\nu\left(\mathcal{E}, L^{2}, r\right)$ on a logarithmic scale, versus the energy $\mathcal{E}$, as calculated from the $N$-body data of the experiment C-2 for $r=0.2$ and for five different values of $L^{2}$ equal to $L^{2}=0,10,20,30,40$ (from top to bottom). The linear right parts of the curves have all similar slopes. The dashed line is the best exponential fit to these curves. The solid line is the best exponential fit of the maximum value of $\nu\left(\mathcal{E}, L^{2}, r\right)$ versus the energy of the maximum $\mathcal{E}=\Psi_{\mathrm{e}}\left(r, L^{2}\right)$. b) The fitting of $\nu\left(\mathcal{E}, L^{2}, r\right)$ (dotted curves) by the model of Eq. (34) (solid curves).

$L^{2}=0,10,20,30$ and 40 (from top to bottom). The following are observed:

1. For fixed $L^{2}$, the $\operatorname{logarithm} \log \left[\nu\left(\mathcal{E}, L^{2}, r\right)\right]$ is with good precision a linear function of the energy $\mathcal{E}$ in the region of energies before the maximum $\mathcal{E}<\Psi_{\mathrm{e}}\left(r, L^{2}\right)$ (dashed curve in Fig. 7a), while it falls quickly to nearly zero for values of the energy beyond the solid line $\mathcal{E}=\Psi_{\mathrm{e}}\left(r, L^{2}\right)$. The linear parts of the curves $\log \left[\nu\left(\mathcal{E}, L^{2}, r\right)\right]$ vs. $\mathcal{E}$, for $\mathcal{E}<\Psi_{\mathrm{e}}\left(r, L^{2}\right)$ and for different values of $L^{2}$ have all similar slopes. Thus we can define an average slope

$$
\beta(r)=\frac{\partial \log \left[\nu\left(\mathcal{E}, L^{2}, r\right)\right]}{\partial \mathcal{E}}, \quad \mathcal{E}<\Psi_{\mathrm{e}}\left(r, L^{2}\right)
$$

which is a constant for one shell. We show below that the values of $\beta(r)$ vary with the radius $r$ of the shell. The parameter $\beta$ can be considered as the inverse of the temperature of the shell.

2. The maxima of $\log \left[\nu\left(\mathcal{E}, L^{2}, r\right)\right]$, for different $L^{2}$, at the energies $\mathcal{E}=\Psi_{\mathrm{e}}\left(r, L^{2}\right)$ are also good linear functions of $\mathcal{E}=\Psi_{\mathrm{e}}\left(r, L^{2}\right)$ (solid line in Fig. 7a). We can define a slope for this linear relation as:

$B(r)=\frac{\partial \log \left[\nu\left(\Psi_{\mathrm{e}}, L^{2}, r\right)\right]}{\partial \Psi_{\mathrm{e}}}$.

The parameter $B(r)$ depends also on the radius $r$ of the shell. In general, the slope $B(r)$ is different from the slope $\beta(r)$. It is explained below that the difference $B(r)-\beta(r)$ is a measure of the radial anisotropy of the velocity ellipsoid within the shell.

\subsection{Model for $\nu\left(\mathcal{E}, L^{2}, r\right)$}

Due to remark (1) above we adopt the following exponential law:

$$
\nu\left(\mathcal{E}, L^{2}, r\right)=A\left(r, L^{2}\right) \mathrm{e}^{\beta(r) \mathcal{E}} \quad \mathcal{E}<\Psi_{\mathrm{e}}\left(r, L^{2}\right),
$$

where $A\left(r, L^{2}\right)$ gives the amplitude of the distribution for a given value of $L^{2}$. The value of the maximum $\nu_{\max }\left(r, L^{2}\right)$ corresponds to the value of the energy $\mathcal{E}=\Psi_{\mathrm{e}}\left(r, L^{2}\right)$, i.e. we have

$\nu_{\max }\left(r, L^{2}\right)=A\left(r, L^{2}\right) \mathrm{e}^{\beta(r) \Psi_{\mathrm{e}}\left(r, L^{2}\right)}$.

On the other hand, according to remark (2) above, the maximum value of $\nu\left(\mathcal{E}, L^{2}, r\right)$ depends exponentially on the energy of the maximum, that is

$\nu_{\max }\left(r, L^{2}\right)=D(r) \mathrm{e}^{B(r) \Psi_{\mathrm{e}}\left(r, L^{2}\right)}$

where $D(r)$ is a parameter depending on the shell radius only. Equating the r.h.s of Eqs. (27) and (28) we obtain

$A\left(r, L^{2}\right)=D(r) \mathrm{e}^{[B(r)-\beta(r)] \Psi_{\mathrm{e}}\left(r, L^{2}\right)}$.

Substituting Eq. (29) into Eq. (26) we obtain the following model for the spherical shell number density function:

$$
\begin{array}{r}
\nu\left(\mathcal{E}, L^{2}, r\right)=D(r) \mathrm{e}^{B(r) \Psi_{\mathrm{e}}\left(r, L^{2}\right)} \mathrm{e}^{\beta(r)\left[\mathcal{E}-\Psi_{\mathrm{e}}\left(r, L^{2}\right)\right]} \\
\mathcal{E}<\Psi_{\mathrm{e}}\left(r, L^{2}\right) .
\end{array}
$$

For the energies $\mathcal{E}>\Psi_{\mathrm{e}}\left(r, L^{2}\right)$ we have a quick fall of the distribution $\nu\left(\mathcal{E}, L^{2}, r\right)$, that is the value of the energy $\mathcal{E}=\Psi_{\mathrm{e}}\left(r, L^{2}\right)$ plays the role of a smooth cut-off for the distribution. To introduce this smooth cut-off, we divide 
the right hand of Eq. (30) with a denominator of the type of the Lynden-Bell distribution function. Thus, the final model for the spherical shell number density function is given by

$\nu\left(\mathcal{E}, L^{2}, r\right)=\frac{D(r) \mathrm{e}^{B(r) \Psi_{\mathrm{e}}\left(r, L^{2}\right)} \mathrm{e}^{\beta(r)\left[\mathcal{E}-\Psi_{\mathrm{e}}\left(r, L^{2}\right)\right]}}{\mathrm{e}^{\beta_{\mathrm{c}}\left[\mathcal{E}-\mu\left(r, L^{2}\right)\right]}+1}$.

where the quantity $\mu$ is defined by

$\mu\left(r, L^{2}\right)=\Psi_{\mathrm{e}}\left(r, L^{2}\right)+\frac{\beta(r)\left[\log \beta(r)-\log \left(\beta_{\mathrm{c}}-\beta(r)\right)\right]}{\beta_{\mathrm{c}}}$

so that the maxima of the quantity $\nu\left(\mathcal{E}, L^{2}, r\right)$ are exactly on the values of the energy $\mathcal{E}=\Psi_{\mathrm{e}}\left(r, L^{2}\right)$, as one can easily verify by differentiating Eq. (31) with respect to $\mathcal{E}$. The quantity $\mu\left(r, L^{2}\right)$ differs from $\Psi_{\mathrm{e}}\left(r, L^{2}\right)$ by a small correction term. If we simply set $\mu\left(r, L^{2}\right)=\Psi_{\mathrm{e}}\left(r, L^{2}\right)$ then the maximum of Eq. (31) is shifted with respect to $\Psi_{\mathrm{e}}\left(r, L^{2}\right)$ by 1 to 2 units of $\mathcal{E}$. This shift is important especially for the spherical shells of large radii, because the distribution $\nu\left(\mathcal{E}, L^{2}, r\right)$ for these radii is narrow in the energy axis (e.g., compare the contours of $\nu\left(\mathcal{E}, L^{2}, r\right)$ in Fig. 6 for $r=0.05$ and for $r=0.3)$. On the other hand, our model is not very sensitive on the choice of the value of the constant $\beta_{\mathrm{c}}$ in Eq. (32), provided that $\beta_{\mathrm{c}} \approx 1$. We set $\beta_{\mathrm{c}}=1$ for all the experiments.

If we substitute Eq. (21) of the effective potential $\Psi_{\mathrm{e}}\left(r, L^{2}\right)$ into Eq. (31) we find

$\nu\left(\mathcal{E}, L^{2}, r\right)=\frac{D(r) \mathrm{e}^{[B(r)-\beta(r)] \Psi_{0}(r)} \mathrm{e}^{\beta(r) \mathcal{E}+[\beta(r)-B(r)] L^{2} / 2 r^{2}}}{\mathrm{e}^{\beta_{\mathrm{c}}\left[\mathcal{E}-\mu\left(r, L^{2}\right)\right]}+1}$,

or, more simply

$\nu\left(\mathcal{E}, L^{2}, r\right)=\frac{C(r) \mathrm{e}^{\beta(r) \mathcal{E}+[\beta(r)-B(r)] L^{2} / 2 r^{2}}}{\mathrm{e}^{\beta_{c}\left[\mathcal{E}-\mu\left(r, L^{2}\right)\right]}+1}$,

where

$C(r)=D(r) \mathrm{e}^{[B(r)-\beta(r)] \Psi_{0}(r)}$.

The parameter $C(r)$ fixes the total mass within the shell.

Equation (34) shows clearly the dependence of the distribution $\nu\left(\mathcal{E}, L^{2}, r\right)$ on the variables $\mathcal{E}$ and $L^{2}$. When $\mathcal{E}<\mu\left(r, L^{2}\right)$ the denominator of Eq. (34) is almost equal to unity. Therefore, Eq. (34) is further simplified to the form

$\nu\left(\mathcal{E}, L^{2}, r\right)=C(r) \mathrm{e}^{\beta(r) \mathcal{E}} \mathrm{e}^{[\beta(r)-B(r)] L^{2} / 2 r^{2}}$.

From Eq. (36) it is clear that the difference $\beta(r)-B(r)$ measures the radial anisotropy at the radius $r$ of the shell. If $\beta(r)-B(r)=0$ the distribution $\nu\left(\mathcal{E}, L^{2}, r\right)$ becomes independent of the angular momentum $L^{2}$, hence the distribution of the velocities is isotropic. If $\beta(r)-B(r)<0$ the distribution $\nu\left(\mathcal{E}, L^{2}, r\right)$ is radially anisotropic, since it is a decreasing function of $L^{2}$, i.e. it favors orbits with $L^{2} \rightarrow 0$.

Figure $7 \mathrm{~b}$ shows the fitting of the function $\nu\left(\mathcal{E}, L^{2}, r\right)$ by the model of Eq. (34) for the best fitting parameters $\beta$,
$B, C$ and $\beta_{\mathrm{c}}=1$. We have examined shells of various radii for all the experiments. We always found good agreement between the model given by Eq. (34) and the $N$-body data for $\nu\left(\mathcal{E}, L^{2}, r\right)$.

\subsection{Fitting of the functions $\beta(r), B(r)$ and $C(r)$}

In order to obtain global fits for all the experiments, the dependence of the parameters $\beta, B, C$ on the radius $r$ has first to be calculated.

The parameters $\beta(r)$ and $B(r)$ are easily calculated from the best fit lines (dashed and solid respectively) similar to those of Fig. 7a, for all values of $r$. In Fig. 8 we show these parameters for all the experiments. Both parameters have similar behavior for all the experiments. As $r \rightarrow 0$ they tend to a constant value $\beta \approx B \approx 0.1$. This means that for all the systems we have isotropy near the center. As $r$ increases, both parameters increase. The physical reason for this is that the temperature (velocity dispersion) decreases as the distance from the center increases. However, the parameter $B(r)$ increases faster than $\beta(r)$. Thus, as we move outwards we have $B(r)>\beta(r)$ which implies radial anisotropy. The behavior of the parameters is unclear near the end of each system, at radii between $r=0.3$ to $r=0.5$, because of the noise of the numerical data used to derive them as best fit parameters. In some cases we see a tendancy of both parameters to stabilize around the values $B \approx 1$ and $\beta \approx 0.3$ respectively. The solid curves shown in Fig. 8 are best fits of the functions $\beta(r)$ and $B(r)$ by a quadratic polynomial.

A remarkable regularity was found when fitting the parameter $C(r)$. Putting $L^{2}=0$ in Eq. (21) we have $\Psi_{\mathrm{e}}\left(r, L^{2}\right)=\Psi_{0}(r)$. Substituting $\mathcal{E}=\Psi_{0}(r)$ in Eq. $(36)$ we obtain

$C(r)=\nu\left(\Psi_{\mathrm{o}}(r), 0, r\right) \mathrm{e}^{-\beta(r) \Psi_{0}(r)}$.

The quantity $\nu\left(\Psi_{\mathrm{o}}(r), 0, r\right)$ gives the number of particles per unit radius, energy and angular momentum which reach the shell of radius $r$ with zero total velocity. We found that in all the experiments the function $\nu\left(\Psi_{\mathrm{o}}(r), 0, r\right)$ can be fitted by exactly the same very simple law given by

$\log \left[\nu_{\text {fit }}\left(\Psi_{\mathrm{o}}(r), 0, r\right)\right]=12.7-0.6\left[\Psi_{0}(0)-\Psi_{0}(r)\right]^{0.5}$

This fitting is shown in Fig. 9. Equation (38) fits nicely the data in the whole range of radii up to a maximum radius where the logarithmic slope of the function $\nu\left(\Psi_{\mathrm{o}}(r), 0, r\right)$ changes abruptly. This radius defines roughly the end of each system.

We do not have a theoretical explanation for this remarkable result. However, Eq. (38) may be related to the distribution of the velocities of the particles in radial orbits which cross the center and have $r$ as their apocenter. This is because the conservation of the energy implies that such velocities are given by $v=\sqrt{2\left[\Psi_{0}(0)-\Psi_{0}(r)\right]}$, i.e. $\sqrt{2}$ times the quantity appearing in the r.h.s. of Eq. (38). 
a) $\mathrm{S}-3$

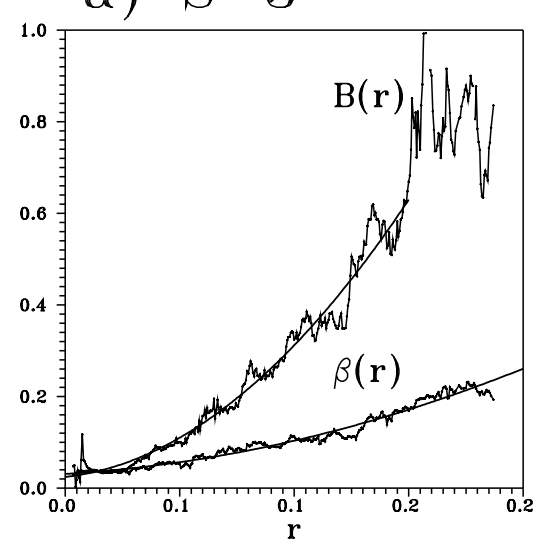

d) $\mathrm{SO}$
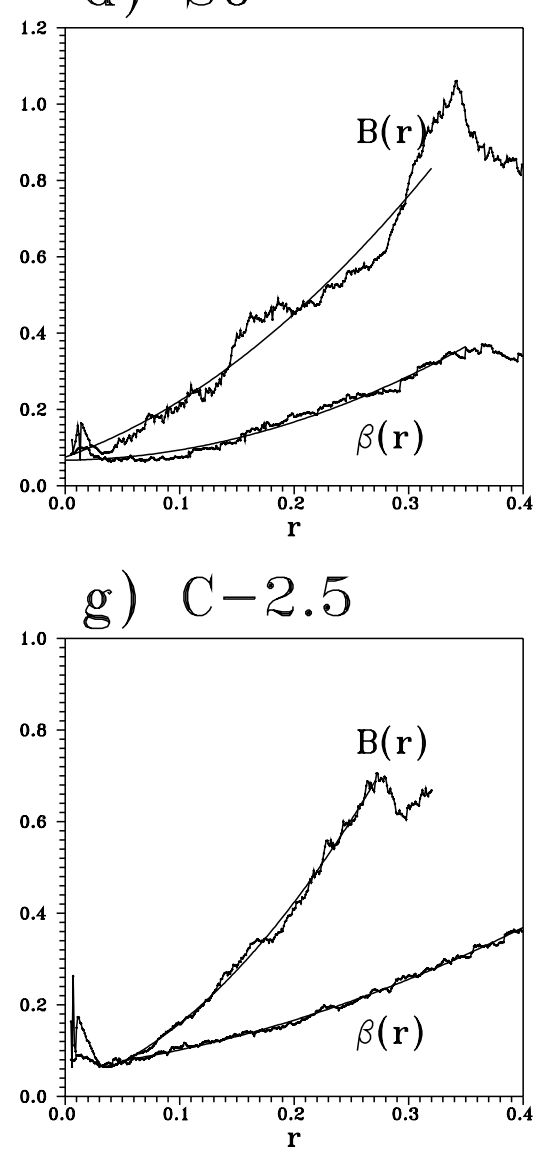

b) $\mathrm{S}-2$

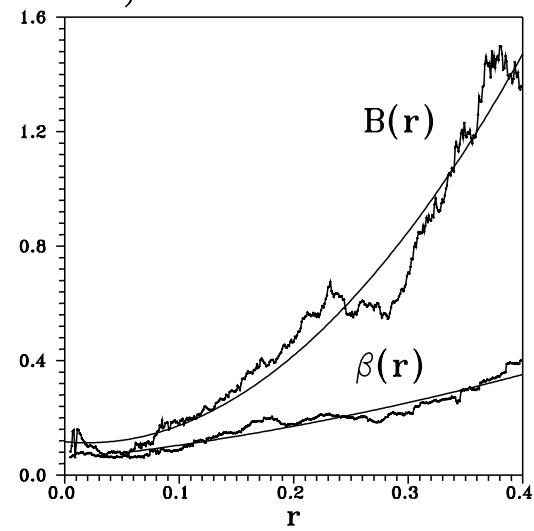

e) $\mathrm{S} 1$

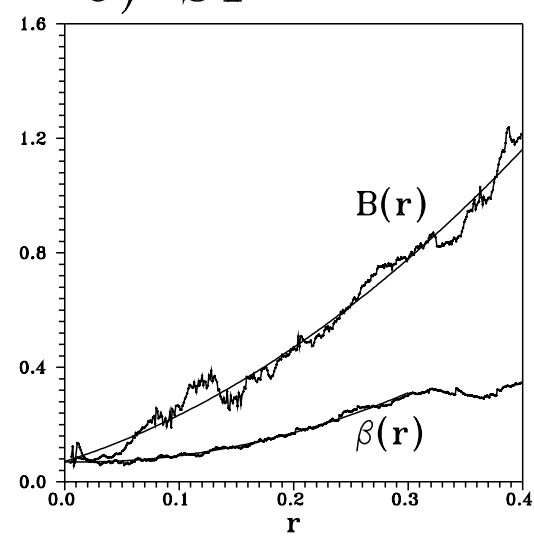

h) $\mathrm{C}-2$

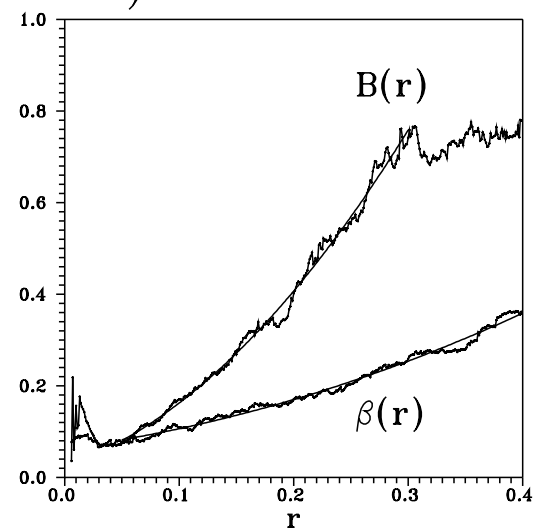

c) $\mathrm{S}-1$

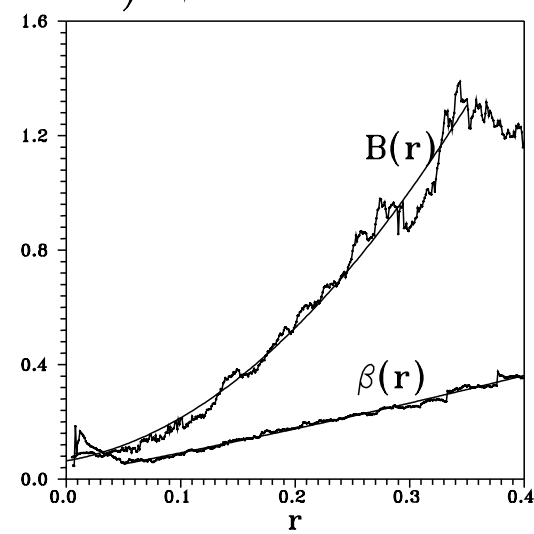

f) $\mathrm{C}-3$

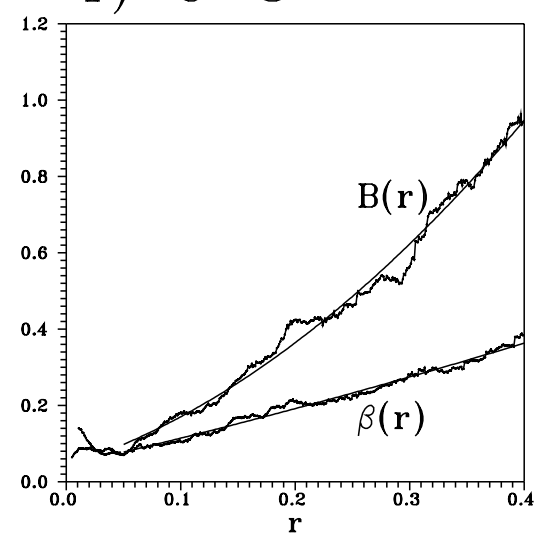

i) $\mathrm{C}-1$

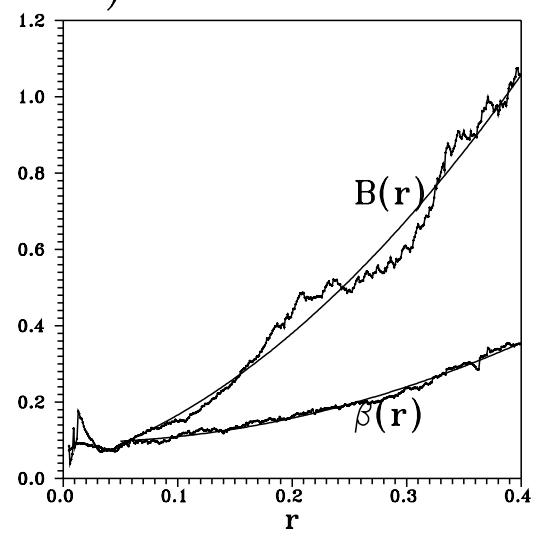

Fig. 8. The fitting parameters $B(r)$ and $\beta(r)$ as functions of $r$ for all the experiments. The solid curves are best fittings by a quadratic polynomial.

\section{Global fitting of the number density and of the distribution function}

The number density $N\left(\mathcal{E}, L^{2}\right)$ of a system is the sum of the spherical shell number density functions for all possible radii, namely

$N\left(\mathcal{E}, L^{2}\right)=\int_{0}^{\infty} \nu\left(\mathcal{E}, L^{2}, r\right) \mathrm{d} r$.
The integral (39) can be evaluated after $\nu\left(\mathcal{E}, L^{2}, r\right)$ has been fitted for each experiment.

In Fig. 10 we show the fitting model $N\left(\mathcal{E}, L^{2}\right)$ produced by the integration (39) compared with the numerical data for all the experiments. The model gives satisfactory fits both for the clumpy and the non clumpy experiments. In particular, the integral (39) reproduces in a natural way both the "core" and the "halo" local maxima of $N\left(\mathcal{E}, L^{2}\right)$ that were introduced by hand in the model of Sect. 4 . 
a) $\mathrm{S}-3$

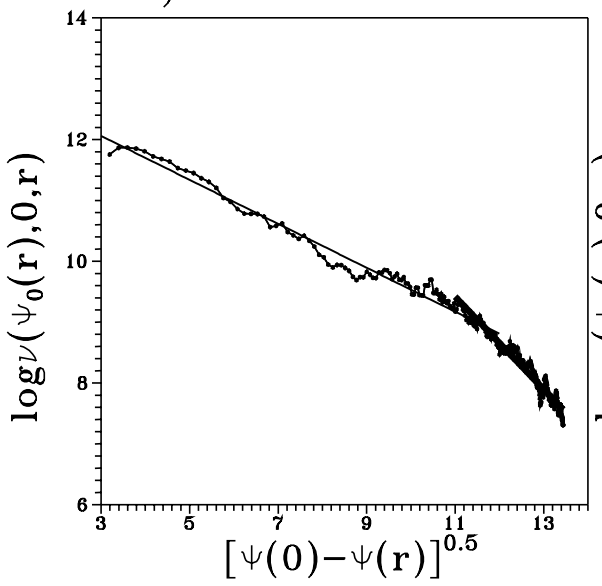

d) $\mathrm{SO}$

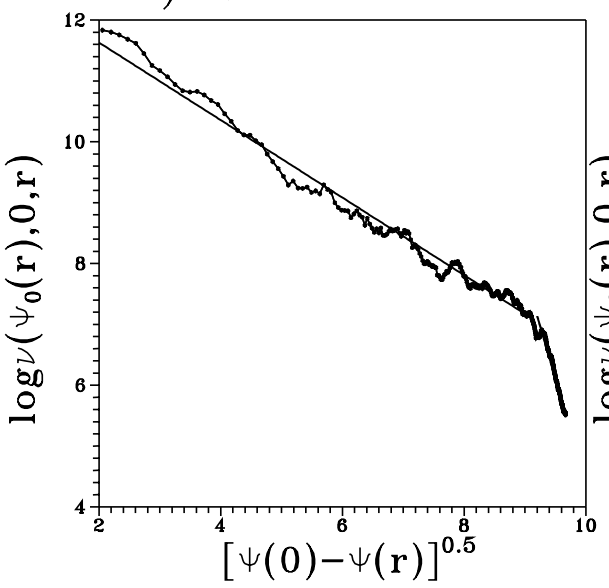

g) $\mathrm{C}-2.5$

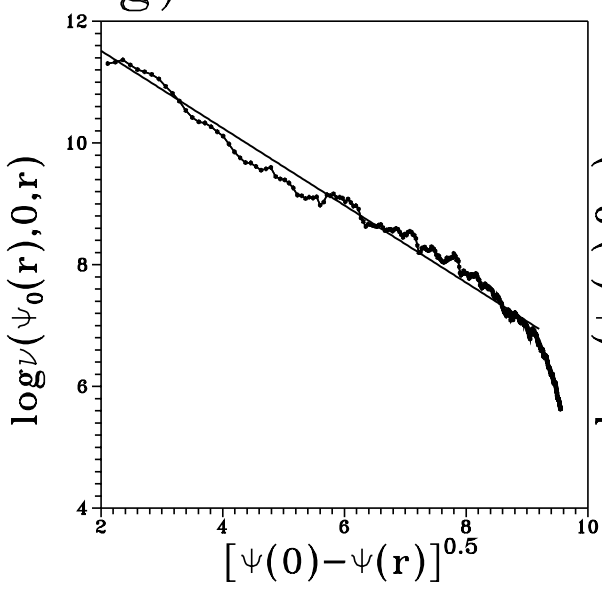

b) $\mathrm{S}-2$

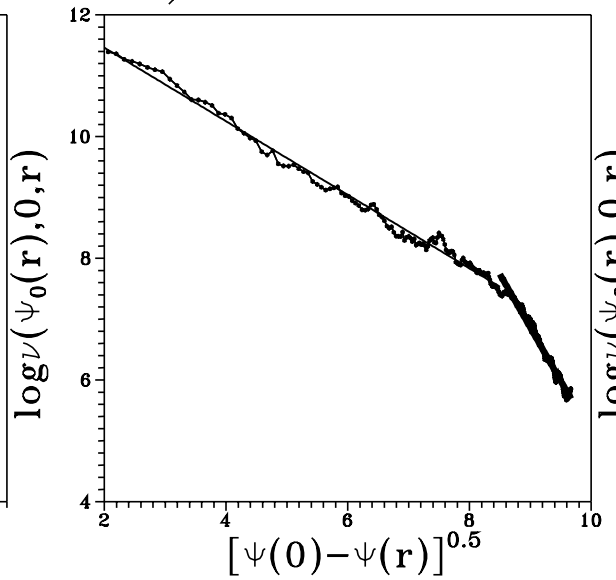

e) $\mathrm{S} 1$

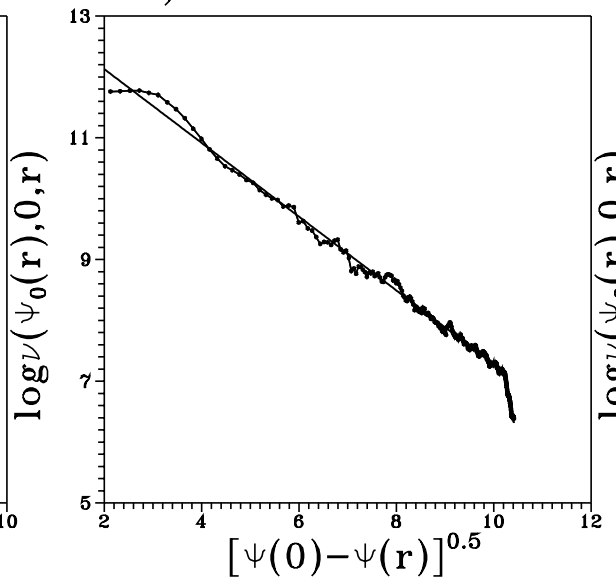

h) $\mathrm{C}-2$

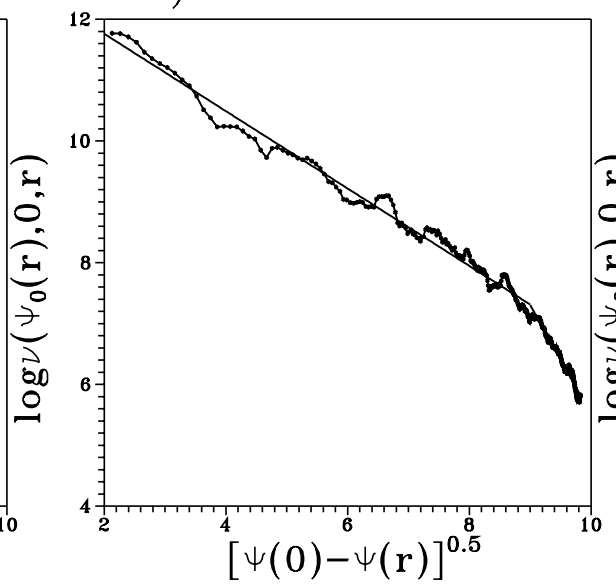

c) $\mathrm{S}-1$

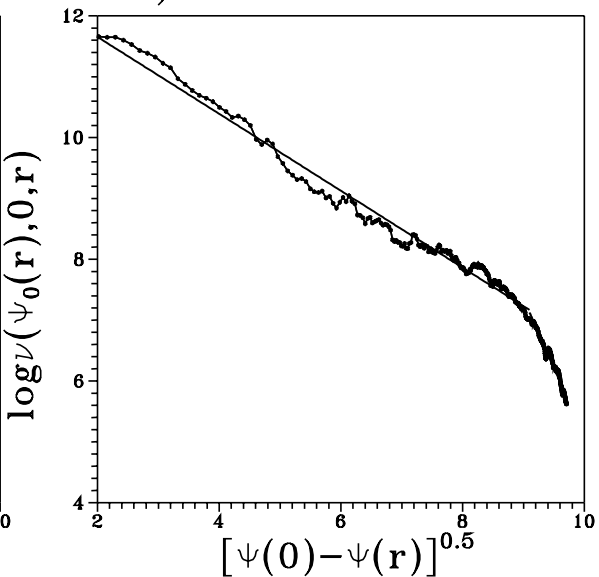

f) $\mathrm{C}-3$

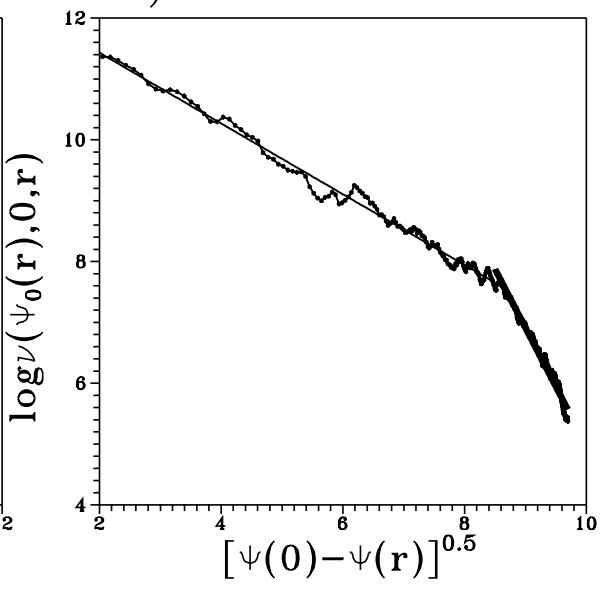

i) $\mathrm{C}-1$

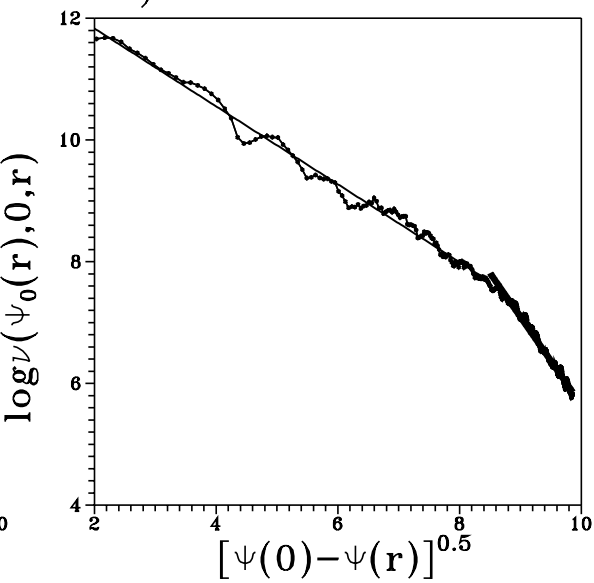

Fig. 9. The fitting parameter $\log \nu\left(\Psi_{0}(r), 0, r\right)$ as a function of $r$ for all the experiments. The solid lines correspond to the universal law given by Eq. (38).

The appearance of these maxima is explained as follows.

As shown in the previous section, the maxima of $\nu\left(\mathcal{E}, L^{2}, r\right)$ are on the lines (Eq. $\left.(20)\right)$

$L^{2}=-2 r^{2}\left[\mathcal{E}-\Psi_{0}(r)\right]$
In Fig. 11 this family of lines is plotted for various values of the radius $r$. It is easy to show that the caustic defined by these lines corresponds to the curve $\mathcal{E}_{\mathrm{c}}\left(L^{2}\right)$ of the circular orbit of the monopole potential term (Eq. (16)).

As $r \rightarrow 0$ the lines become almost horizontal and the function $\nu\left(\mathcal{E}, L^{2}, r\right)$ on these lines increases from left to (40) right. These almost horizontal lines produce a maximum 

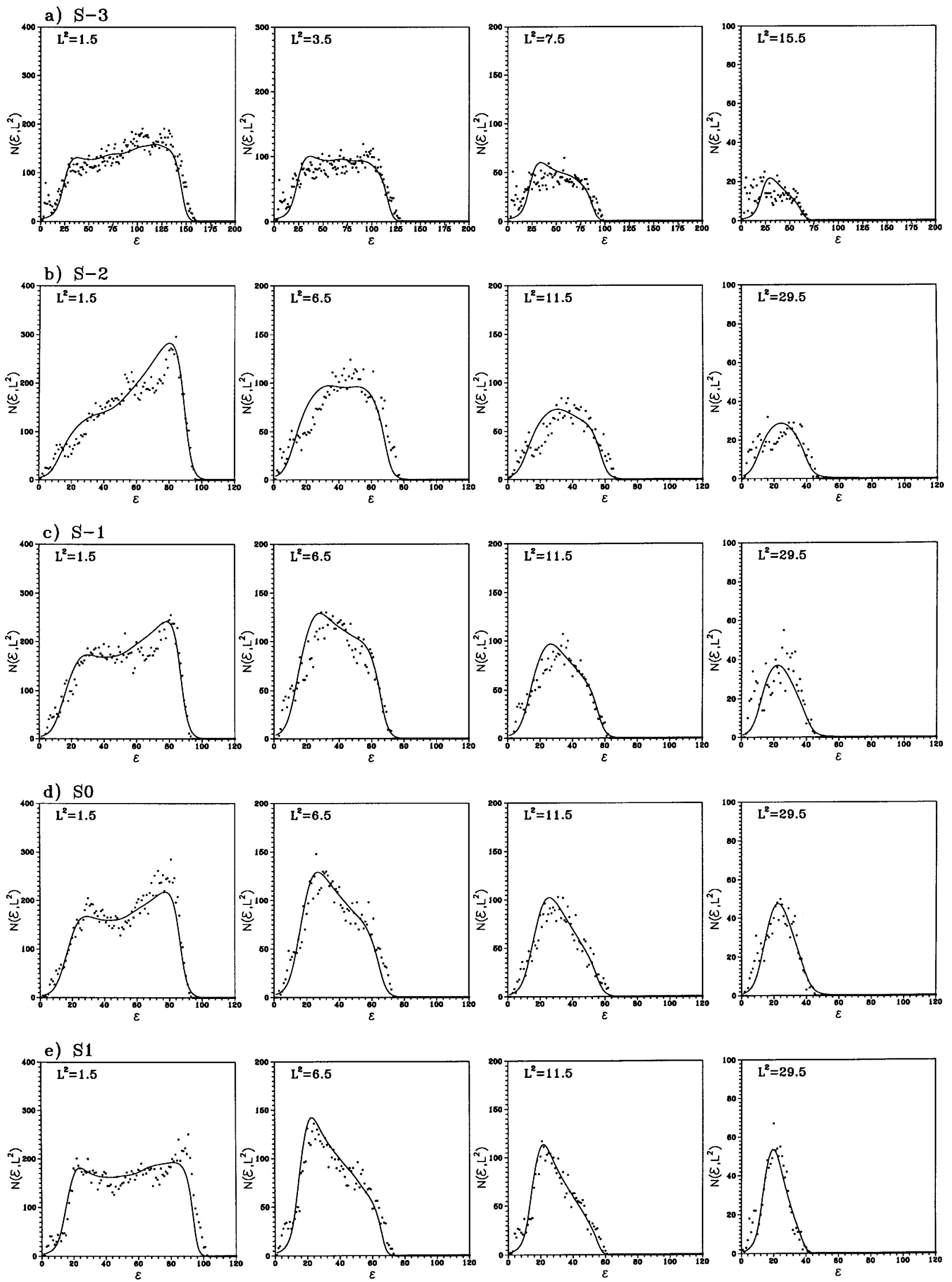

Fig. 10. The fitting of $N\left(\mathcal{E}, L^{2}\right)$ (dots) by the model of Eq. (39) (solid curve) for all the experiments. For each experiment, the fitting is shown along the energy axis, for four different slices of constant angular momentum $L^{2}$. 

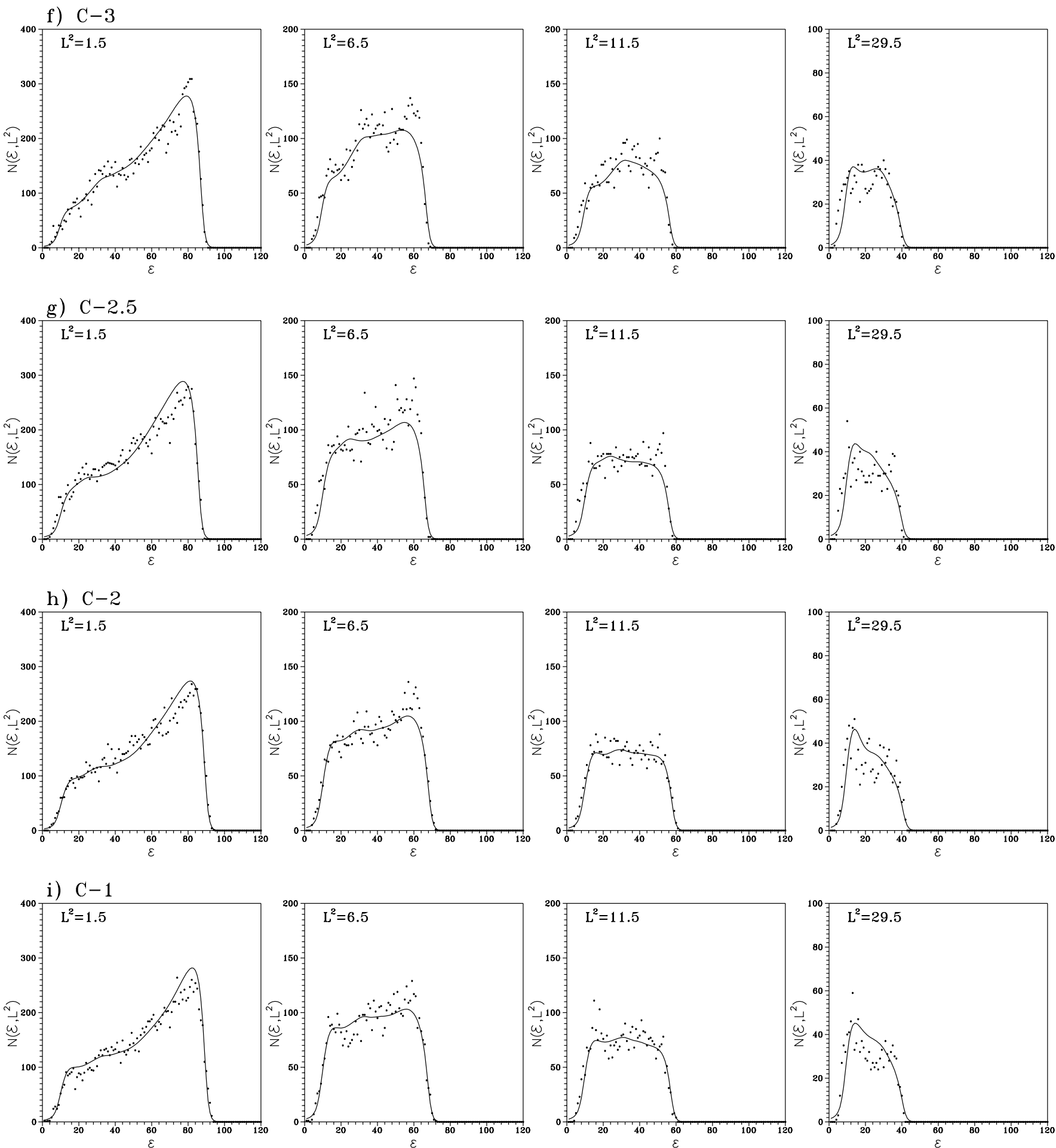

Fig. 10. continued.

in the bottom right side of Fig. 11 which is the "core" maximum of Sect. 4.

However, as $r$ increases the slope of the lines of maximum increases and the lines tend to become vertical. The function $\nu\left(\mathcal{E}, L^{2}, r\right)$ along the vertical lines increases from up down (as $L^{2} \rightarrow 0$ ). These lines produce the "halo" maximum of Sect. 4 .
Thus we see that there is no fundamental distinction between the core and the halo maxima. They are both produced by the same statistics given by Eq. (34) for the spherical shell number density function $\nu\left(\mathcal{E}, L^{2}, r\right)$.

As analyzed in Sect. 2, we can use the global fitting of the number density function $N\left(\mathcal{E}, L^{2}\right)$ in order to produce a global fitting of the coarse grained distribution function given by Eq. (6). To do so, we need to calculate the 


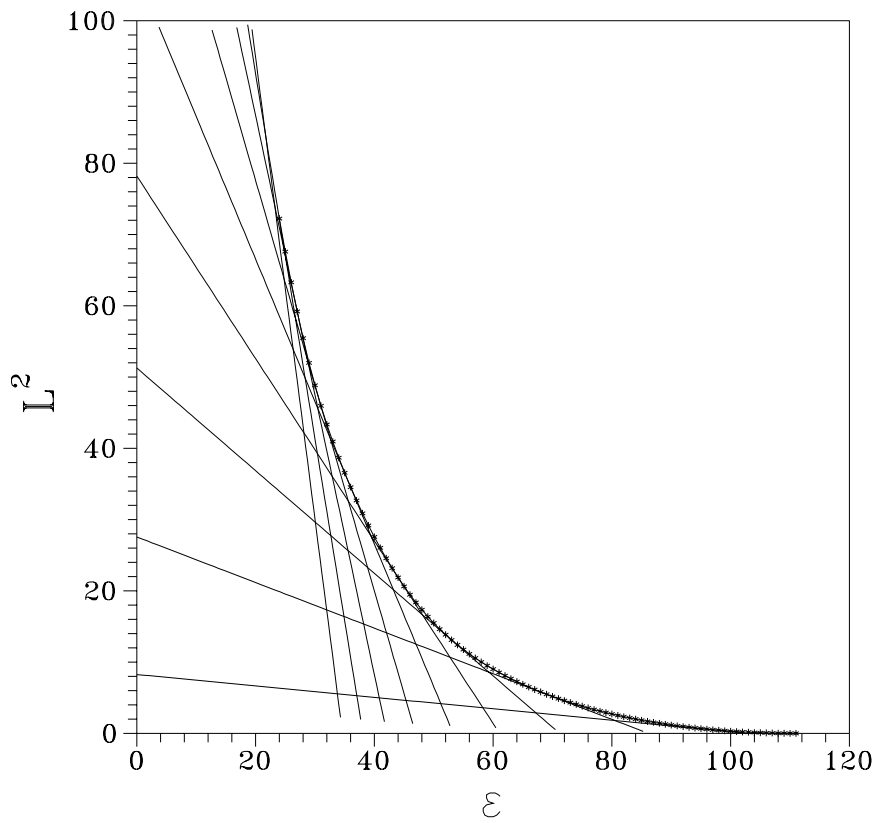

Fig. 11. Nine lines of Eq. (40) for the values of $r$ equal to $r=$ $0.02,0.04, \ldots, 0.18$, for the experiment $\mathrm{C}-2$. The dotted curve is the caustic of these lines, which corresponds to the curve of the circular orbit $\mathcal{E}_{\mathrm{c}}\left(L^{2}\right)$.

density of states function $\omega\left(\mathcal{E}, L^{2}\right)$ given by Eqs. (4) and (5). In all the experiments we found that $\omega\left(\mathcal{E}, L^{2}\right)$ depends weakly on $L^{2}$. For a fixed value of the energy $\mathcal{E}$, $\omega\left(\mathcal{E}, L^{2}\right)$ varies by less than $10 \%$ in the permissible interval of values of $L^{2}$ between $L^{2}=0$ and $L^{2}=L_{\mathrm{c}}^{2}(\mathcal{E})$, where $L_{\mathrm{c}}^{2}(\mathcal{E})$ denotes the angular momentum of the circular orbit with energy $\mathcal{E}$ under the influence of the monopole potential term. This situation appears in many galactic spherical potentials (see e.g., Palmer 1994).

As regards the dependence of $\omega\left(\mathcal{E}, L^{2}\right)$ on $\mathcal{E}$, we found that it is very well represented by the law $\omega \propto \mathcal{E}^{-3 / 2}$ (this law is exact for the Keplerian and isochrone potentials). The proportionality constant was found for each experiment by a linear best fit of $\omega$ vs. $\mathcal{E}$ on a $\log -\log$ scale.

In order to test the quality of the fitting of the coarse grained distribution function by the above procedure, we calculated several moments of this distribution function and compared them with the numerical data. In Fig. 12 the fitting is shown for the density function

$\rho(r)=4 \pi \int_{0}^{\sqrt{2 \Psi_{0}(r)}} \mathrm{d} v_{\mathrm{r}} \int_{0}^{\sqrt{2 \Psi_{0}(r)-v_{\mathrm{r}}^{2}}} F v_{\mathrm{t}} \mathrm{d} v_{\mathrm{t}}$

for all the experiments. The "numerical" density function is evaluated by counting the number of particles within a spherical shell of radius $r$ and width $\mathrm{d} r$ divided by the volume of the shell. This is a "spherically averaged" function, since the systems are in fact triaxial and the density $\rho$ has not constant value at all points within such a spherical shell. However, the main interest in these diagrams is on the logarithmic slope of $\rho$ as a function of $r$, which defines an average radial profile for $\rho$. The inner $\approx 7 \%$ of the mass of each system is within the softening radius of the $N$-body code, and we cannot recover the slope of the profile for this mass fraction. The slope is $\approx-1$ for the radii corresponding to mass fractions between $7 \%$ and $20 \%$. From there on, the slope turns gradually to -2 until the half mass radius, and to -3 for larger distances. At the end of each system we obtain asymptotically the slope -4 .

A second parameter of interest is the anisotropy parameter

$\beta_{\mathrm{a}}(r)=1-\frac{\sigma_{\mathrm{t}}^{2}(r)}{2 \sigma_{\mathrm{r}}^{2}(r)}$

(e.g., Binney \& Tremaine 1987), where $\sigma_{\mathrm{r}}^{2}(r)$ and $\sigma_{\mathrm{t}}^{2}(r)$ are the velocity dispersions in the radial and transverse direction of motion given by

$\sigma_{\mathrm{r}}^{2}(r)=\frac{1}{\rho(r)} \int_{0}^{\sqrt{2 \Psi_{0}(r)}} v_{\mathrm{r}}^{2} \mathrm{~d} v_{\mathrm{r}} \int_{0}^{\sqrt{2 \Psi_{0}(r)-v_{\mathrm{r}}^{2}}} F v_{\mathrm{t}} \mathrm{d} v_{\mathrm{t}}$

and

$\sigma_{\mathrm{t}}^{2}(r)=\frac{1}{\rho(r)} \int_{0}^{\sqrt{2 \Psi_{0}(r)}} \mathrm{d} v_{\mathrm{r}} \int_{0}^{\sqrt{2 \Psi_{0}(r)-v_{\mathrm{r}}^{2}}} F v_{\mathrm{t}}^{3} \mathrm{~d} v_{\mathrm{t}}$

respectively. In Fig. 13 we show the fitting of the anisotropy parameter for all the experiments. There is again good agreement between the model and the numerical data. To give some error estimates, the largest errors found were $7 \%$ for the fitting of the $\rho(r)$ profiles and $10 \%$ for the fitting of the $\beta_{\mathrm{a}}(r)$ profiles.

\section{Theoretical discussion}

Given the good quality of the fitting provided by the model of the spherical shell number density function given by Eq. (34), we discuss now a possible physical interpretation for this model.

One can clasify existing models of the distribution function of stellar systems in two main categories.

1) Models of maximum entropy in phase space. The main feature of such models is the fact that the coarse grained distribution function depends exponentially on the energy and possibly on the angular momentum. Namely, these models have essentially the form:

$F \propto \exp \left(\beta \mathcal{E}-\beta^{\prime} L^{2}\right)$.

The well known "isothermal models" of King $(1962,1965)$ and of Michie (1963), as well as the non degenerate limit of Lynden-Bell (1967) model belong to this category.

Such models are derived theoretically from the request that a suitably defined functional of $F$, corresponding to the entropy of the system, is maximized. The maximization of the entropy is subject to one or more constraints that are also expressed in terms of functionals of $F$. For example, the isothermal model $F \propto \exp (\beta \mathcal{E})$ is derived from the request that the usual Boltzmann entropy

$S=-\int F \ln F \mathrm{~d}^{6} \mu$ 


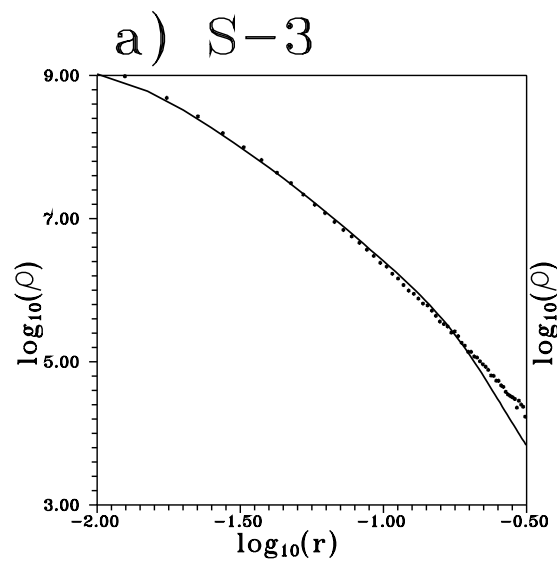

b) $S-2$

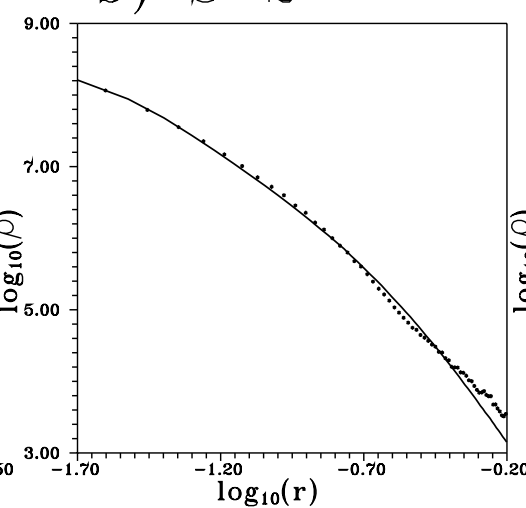

e) $\mathrm{S} 1$

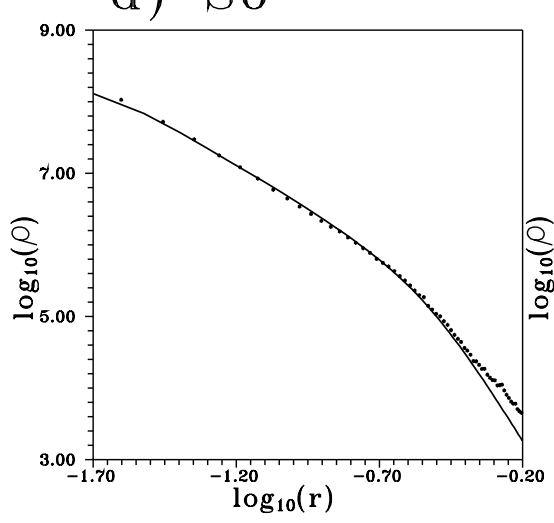

g) $S-2.5$

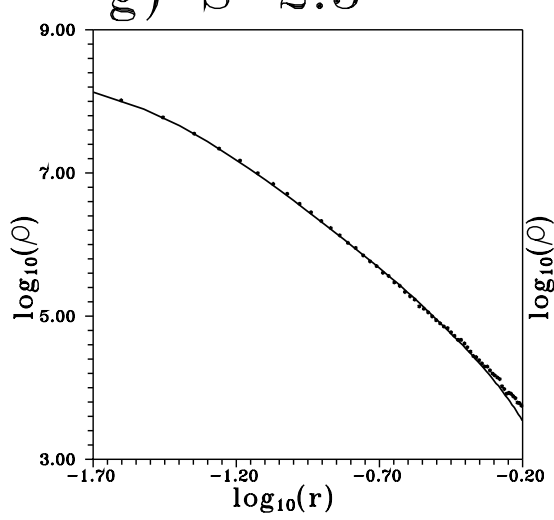

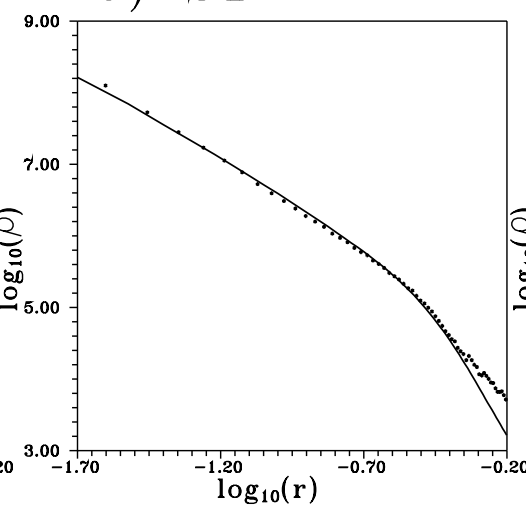

h) $\mathrm{C}-2$

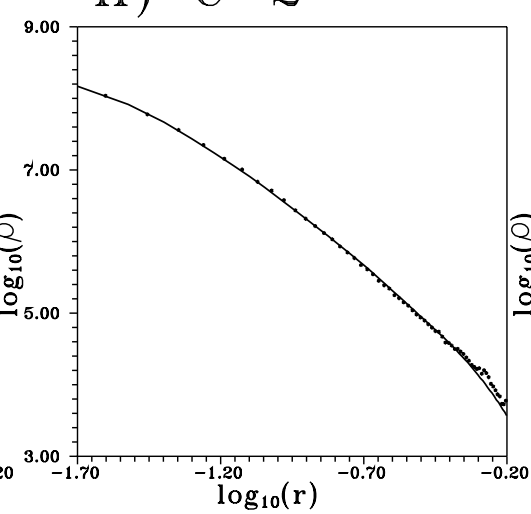

c) $\mathrm{S}-1$

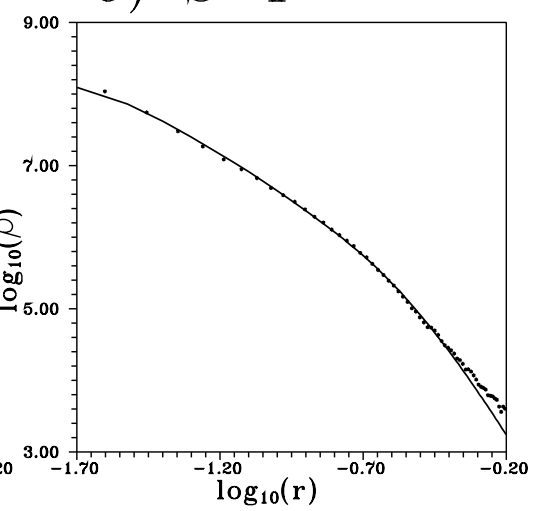

f) $\mathrm{C}-3$

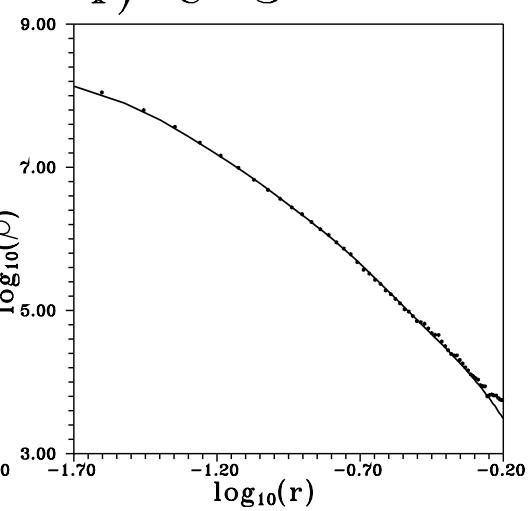

i) $\mathrm{C}-1$

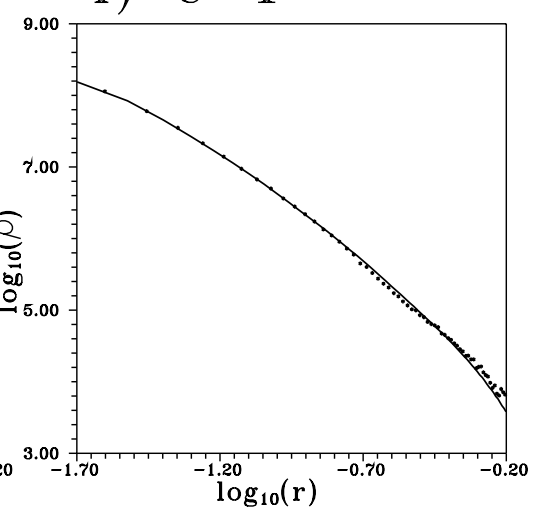

Fig. 12. The fitting of the radial profile of the density (dots) by the model (41) (solid curves), where $F$ is calculated from the spherical shell number density model, for all the experiments.

is maximized under the constraints of constant total mass

$M=\int F \mathrm{~d}^{6} \mu$

and of constant total energy

$$
\begin{aligned}
\mathcal{E}_{\mathrm{tot}} & =-E_{\mathrm{kin}}-E_{\mathrm{dyn}} \\
& =-\frac{1}{2} \int F \boldsymbol{v}^{2} \mathrm{~d}^{6} \mu+\frac{G}{2} \iint \frac{F F^{\prime}}{\left|\boldsymbol{r}-\boldsymbol{r}^{\prime}\right|} \mathrm{d}^{6} \mu \mathrm{d}^{6} \mu^{\prime} .
\end{aligned}
$$

Variants of the isothermal model are derived either by changing the expression for the entropy (e.g., Tremaine et al. 1986), or by considering additional constraints such as the conservation of the total angular momentum (e.g.,
Shu 1969). The constraints appear as constants (e.g., normalization or temperature constant) of the final distribution function, that correspond to the Lagrange multipliers of the Euler maximization of the entropy.

The key point in the "Boltzmann type" statistics is the way by which the expression for the entropy is obtained. This is done by partitioning the usual phase spase in cells that are given equal a priori probabilities of occupation. Then one counts the number of different microstates that correspond to one set of occupation numbers of all the cells (macrostate). The logarithm of the number of microstates that correspond to the same macrostate defines the entropy for that particular macrostate of the system. 
a) $\mathrm{S}-3$

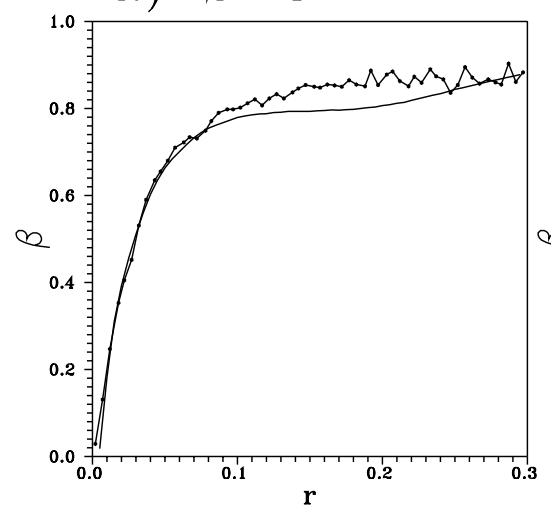

d) $\mathrm{S} 0$

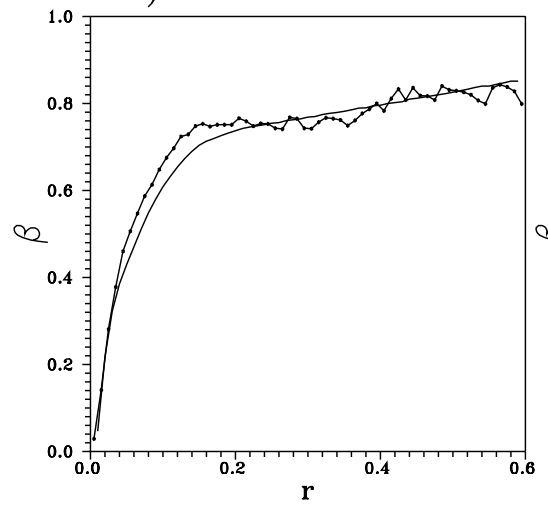

g) $\quad C-2.5$

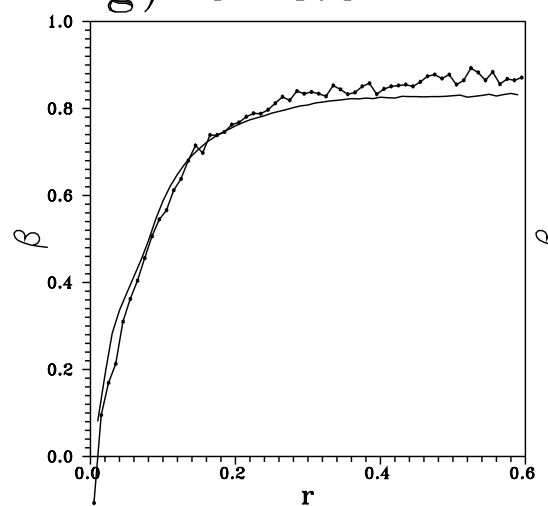

b) $S-2$

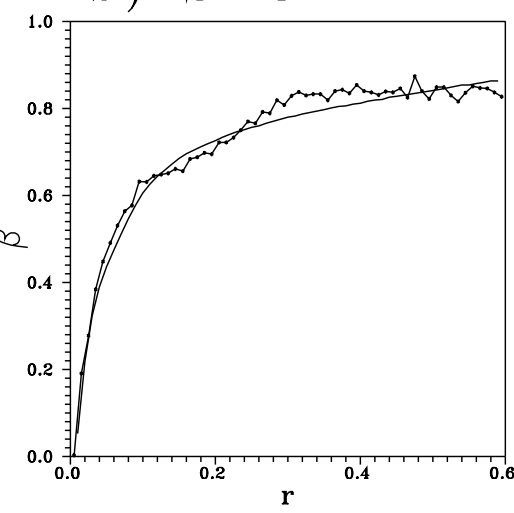

e) S1

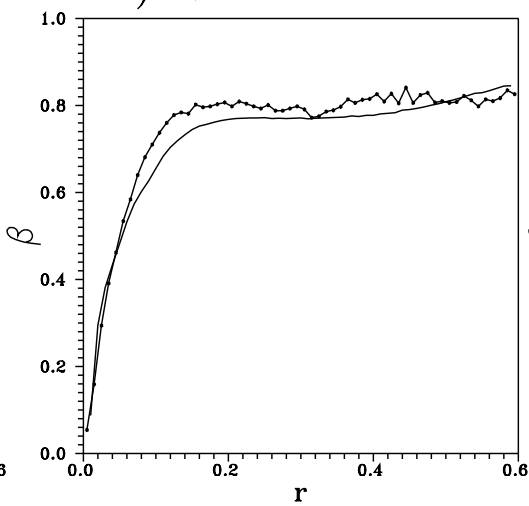

h) $\mathrm{C}-2$

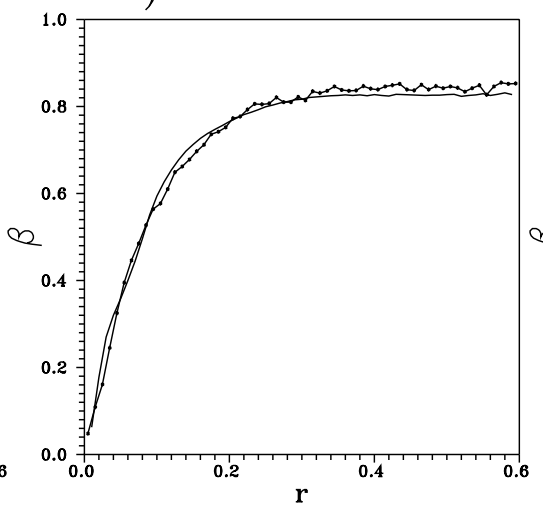

c) $\mathrm{S}-1$

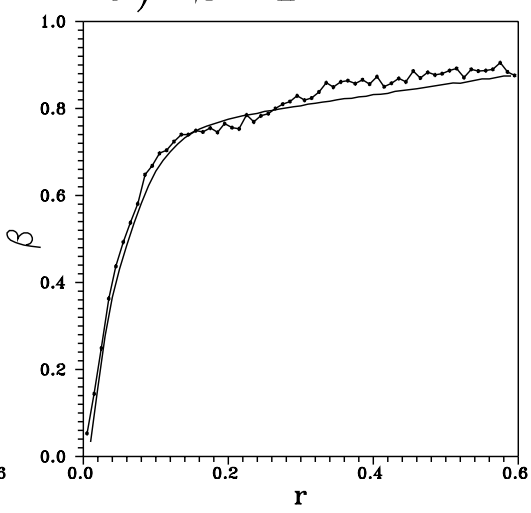

f) $\mathrm{C}-3$

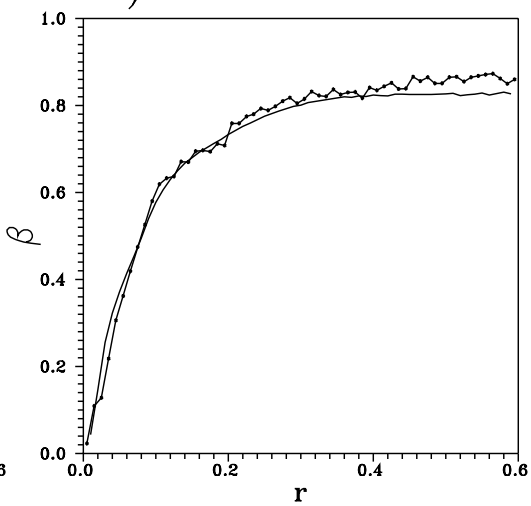

i) $\mathrm{C}-1$

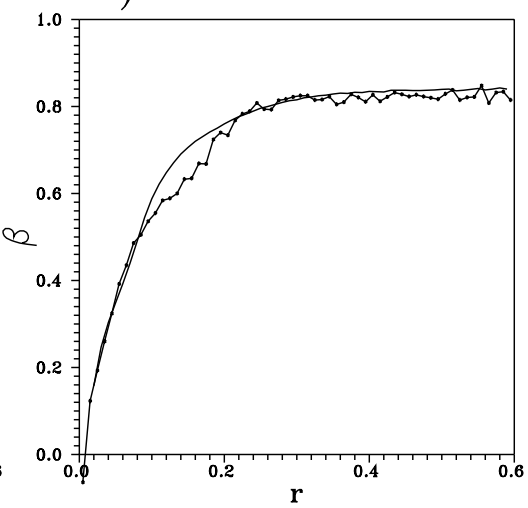

Fig. 13. The fitting of the radial profile of the anisotropy parameter (dots) by the model of Eqs. (42)-(44) (solid curves), where $F$ is calculated from the spherical shell number density model.

2) Models of maximum entropy in the $\left(\mathcal{E}, L^{2}\right)$ space. An example of such models is the spherical Stiavelli-Bertin (1985, 1987) model given by:

$\left.F\left(\mathcal{E}, L^{2}\right)=A \mathcal{E}^{3 / 2} \exp \left[\beta \mathcal{E}-\beta^{\prime} L^{2}\right)\right]$

The difference of this model from the generalised Boltzmann model given by Eq. (45) is the factor $\mathcal{E}^{3 / 2}$ by which the exponential of the distribution function is multiplied. As we saw this factor corresponds to the inverse of the density of states function $\omega\left(\mathcal{E}, L^{2}\right)$. If we multiply
$F$ with $\omega$, we obtain the number density function which is now the function that has the simple exponential form $\left.N\left(\mathcal{E}, L^{2}\right) \propto \exp \left[\beta \mathcal{E}-\beta^{\prime} L^{2}\right)\right]$.

Thus, $N$ rather than $F$ is a function depending exponentially on the energy (and possibly on the angular momentum). Stiavelli \& Bertin (1987) have shown that this model can be derived from a partitioning of the $\left(\mathcal{E}, L^{2}\right)$ space, rather than the phase space, in cells of equal a priori probabilities. In particular, the entropy has the form

$S=-\int N\left(\mathcal{E}, L^{2}\right) \ln N\left(\mathcal{E}, L^{2}\right) \mathrm{d} \mathcal{E} \mathrm{d} L^{2}$ 
which implies non equal a priori probability (and non equal volume) of the cells of the usual phase space (Stiavelli \& Bertin 1987; see also Tremaine 1987; Tremaine et al. 1989).

To the best of our knowledge the first such model of the form $N \propto \exp (\beta \mathcal{E})$ was proposed by Binney (1982). Binney found this model by calculating, through the Eddington (1916) inversion formula, the spherical isotropic distribution function that corresponds to the $r^{1 / 4}$ surface brightness profile (de Vaucouleurs 1948).

It is of interest to see which of the two types of statistics is supported by the $N$-body simulations.

As shown in Sect. 6, the spherical shell number density function $\nu\left(\mathcal{E}, L^{2}, r\right)$ is fitted nicely by our formula of Eq. (36)

$\nu\left(\mathcal{E}, L^{2}, r\right) \approx C(r) \exp \left[\beta(r) \mathcal{E}+(\beta(r)-B(r)) L^{2} / 2 r^{2}\right]$.

This formula is the same as Eq. (50) of the Stiavelli-Bertin model if we replace $N$ by our local function $\nu$ for spherical shells, and if we set $\beta \equiv \beta(r)$ and $\beta^{\prime} \equiv-(\beta(r)-B(r)) / 2 r^{2}$. The difference between our model and the Stiavelli-Bertin model is in the value of the Lagrange multipliers. Namely, in the Stiavelli-Bertin models the Lagrange multipliers have constant values $\beta$ and $\beta^{\prime}$ that are global, i.e. the same for all spherical shells within a system. On the contrary, in our model the Lagrange multipliers are functions of the radius $r$. A possible physical interpretation of this fact is the different level of mixing in phase space as we move from the center of a system outwards. This makes natural the assumption that the "constants" (e.g., the temperature) appearing on the expression for $N$ should in fact be functions of the distance $r$ from the center. For example, as shown in Fig. 8, the temperature (inverse of $B$ ) decreases as the distance from the center increases. Such differences are introduced in a simple way in our models which consider the distribution $\nu\left(\mathcal{E}, L^{2}, r\right)$ of the particles within spherical shells of different distances from the center.

\section{Conclusions}

In this paper we study $N$-body gravitational systems at equilibrium, after a violent relaxation process. The systems are produced from clumpy and non-clumpy initial conditions imitating hierarchical clustering in an expanding universe. The main goal is to construct coarse grained spherical anisotropic distribution function models that fit adequately the numerical data of the $N$-body systems at equilibrium.

The main new results are the following:

1. We propose a method to obtain coarse-grained distribution functions for stellar systems. This is achieved by partitioning the phase space in terms of the invariant tori of an "unperturbed" spherical system of which the potential is the spherical part of the potential of the triaxial system. We fit separately the number density function $N\left(\mathcal{E}, L^{2}\right)$ and the phase space volume function $\omega\left(\mathcal{E}, L^{2}\right)$ on the plane $\left(\mathcal{E}, L^{2}\right)$.
2. We present $N$-body experiments of violent relaxation leading to triaxial systems from non-clumpy and clumpy cosmological initial conditions. All the experiments with non clumpy initial conditions are subject to the radial orbit instability and lead to E5-E6 systems at equilibrium. On the other hand, the experiments with clumpy initial conditions lead to systems with moderate triaxiality (E2-E3) at equilibrium.

3. All the models have a similar structure of the histogram $N\left(\mathcal{E}, L^{2}\right)$ that is called a "core and halo" structure, following the terminology of Voglis (1994). The model of Voglis (1994) can fit the function $N\left(\mathcal{E}, L^{2}\right)$ for the experiments with non-clumpy initial conditions. For the experiments with clumpy initial conditions, $N\left(\mathcal{E}, L^{2}\right)$ has a complicated form.

4. The key result is in Sect. 5. We construct a more general model that fits all the experiments, clumpy and non-clumpy, by defining a "spherical shell number density function" $\nu\left(\mathcal{E}, L^{2}, r\right)$ for the particles that are instantaneously inside a shell of radius $r$. This function has a particularly simple form given by Eq. (34). The maximum of $\nu\left(\mathcal{E}, L^{2}, r\right)$, for given $r$, is on a line of the plane $\left(\mathcal{E}, L^{2}\right)$ on which $r$ is the pericenter or apocenter of motion under the monopole potential term.

5. The function $\nu\left(\mathcal{E}, L^{2}, r\right)$ falls exponentially along both the energy and the angular momentum axis. The relevant constants, $\beta$, and $B$ measure the mean temperature and anisotropy of the velocity ellipsoid respectively. These constants, together with the amplitude of $\nu\left(\mathcal{E}, L^{2}, r\right)$, are functions of the radius $r$. For small $r \rightarrow 0$, the line of maximum is parallel to the energy axis and it naturally produces the "core" population. For $r \rightarrow \infty$, the line of maximum turns almost perpendicularl to the energy axis, and it produces the "halo" population. Therefore we explain theoretically the existence of both populations, which in fact correspond to the same statistics given by the superposition of the functions $\nu\left(\mathcal{E}, L^{2}, r\right)$ for all $r$.

6. The function $\omega\left(\mathcal{E}, L^{2}\right)$ is almost independent of $L^{2}$ and it is well approximated by $\omega \propto \mathcal{E}^{-3 / 2}$. Using this and the above model $\nu\left(\mathcal{E}, L^{2}, r\right)$, we calculate a coarse grained distribution function for all the experiments, and we test how well it fits spherically averaged density and anisotropy parameter profiles. The slopes of the density profiles are produced with good precision. The largest noticed deviations between the model and the numerical data are of order $7 \%$ in the density profiles and $10 \%$ in the anisotropy profiles.

7. We finally discuss the relevance of the new models proposed in this paper to well-known models based on the maximization of the entropy in either the usual phase space or the $\left(\mathcal{E}, L^{2}\right)$ space. In the former case, we have typically $F \propto \exp (\beta \mathcal{E})$, while in the latter case we have $N \propto \exp (\beta \mathcal{E})$. Our models favor the latter statistics, but for the function $\nu$ rather than $N$. Our model for $\nu$ is similar to the Stiavelli-Bertin model, but with the difference that the relevant Lagrange multipliers have values depending on the radius $r$. This may reflect the 
different degree of mixing and organisation of the motion at different distances from the center.

Acknowledgements. We thank Drs. Allen, Palmer, Papaloizou and Hernquist for the offer of their codes. Fruitful discussions with Prof. G. Contopoulos are gratefully acknowledged. This research was supported in part by the Research Committee of the Academy of Athens (contract 200/409). C. E. received support by the Greek State Scholarships Foundation (I.K.Y.).

\section{References}

Aguilar, L. A., \& Merritt, D. 1990, ApJ, 354, 33

Allen, A. J., Palmer, P. L., \& Papaloizou, J. 1990, MNRAS, 242,576

Barnes, J., Goodman, J., \& Hut, P. 1986, ApJ, 300, 112

Binney, J. 1982, MNRAS, 200, 951

Binney, J., \& Tremaine, S. 1987, Galactic dynamics (Princeton University Press)

Contopoulos, G., Efthymiopoulos, C., \& Voglis, N. 2001, Cel. Mech. Dyn. Astron., 78, 243

de Vaucouleurs, G. 1948, Ann. d'Astrophys., 11, 247

Eddington, A. 1916, MNRAS, 76, 572

Fridman, A. M., \& Polyachenko, V. L. 1984, Physics of Gravitating Systems (Springer, New York)
Hernquist, L. 1987, ApJS, 64, 715

King, I. 1962, AJ, 67, 471

King, I. 1965, AJ, 70, 376

Lynden-Bell, D. 1967, MNRAS, 136, 101

Merritt, D. 1999, PASP 111, 756, 129

Merritt, D., Tremaine, S., \& Johnstone, D. 1989, MNRAS, 236, 829

Michie, R. W. 1963, MNRAS, 125, 127

Ogorodnikov, K. F. 1965, Dynamics of Stellar Systems (Pergamon, Oxford)

Palmer, P. L. 1994, Stability of Collisionless Stellar Systems (Kluwer Academic Publishers, The Netherlands)

Palmer, P. L., \& Papaloizou, J. 1987, MNRAS, 224, 1043

Palmer, P. L., \& Voglis, N. 1983, MNRAS, 205, 543

Papaphilippou, Y., \& Laskar, J. 1998, A\&A, 243, 1043

Polyachenko, V. L., \& Shukhman, I. G. 1981, SvA, 25, 533

Shu, F. H. 1969, ApJ, 158, 505

Stiavelli, M., \& Bertin, G. 1985, MNRAS, 217, 735

Stiavelli, M., \& Bertin, G. 1987, MNRAS, 229, 61

Tremaine, S. 1987, in IAU Symp. 127, Structure and Dynamics of Elliptical Galaxies, ed. T. de Zeeuw (Reidel, Dordrecht), 367

Tremaine, S., Hénon, M., \& Lynden-Bell, D. 1986, MNRAS, 219,285

Voglis, N. 1994, MNRAS, 267, 379

Zel'dovich, Ya. 1970, A\&A, 5, 89 